Revue d'histoire de l'Amérique française

REVUE D.HISTOIRE DE L'AMÉRIQUE FRANÇAISE

\title{
Pour une méso-histoire du XIXe siècle canadien
}

\section{Gilles Paquet et Jean-Pierre Wallot}

Volume 33, numéro 3, décembre 1979

URI : https://id.erudit.org/iderudit/303794ar

DOI : https://doi.org/10.7202/303794ar

Aller au sommaire du numéro

Éditeur(s)

Institut d'histoire de l'Amérique française

ISSN

0035-2357 (imprimé)

1492-1383 (numérique)

Découvrir la revue

Citer cet article

Paquet, G. \& Wallot, J.-P. (1979). Pour une méso-histoire du XIXe siècle canadien. Revue d'histoire de l'Amérique française, 33(3), 387-425.

https://doi.org/10.7202/303794ar d'utilisation que vous pouvez consulter en ligne.

https://apropos.erudit.org/fr/usagers/politique-dutilisation/ 


\title{
POUR UNE MÉSO-HISTOIRE DU XIXe SIÈCLE CANADIEN*
}

\author{
GILLES PAQUET \\ Université Carleton \\ JEAN-PIERRE WALLOT \\ Université de Montréal
}

The fox knows many things but the

hedgehog knows one big thing.

(Archilochus)

\section{INTRODUCTION}

Au cours de la dernière décennie, une véritable métamorphose s'est opérée au sein de l'historiographie canadienne. La multiplication des historiens professionnels, leur spécialisation et leur intérêt croissant pour les études transdisciplinaires ont contribué à l'exploration d'avenues neuves et au surgissement d'histoires nouvelles, différentes et plus ambitieuses. Plus encore, les historiens canadiens ont commencé à chercher les moyens de combiner ces différentes histoires en une étude de la société globale comme telle. Démarche qui correspond d'ailleurs à un cheminement analogue dans toutes les sciences humaines ${ }^{1}$.

La réflexion sur l'éventail des possibles qu'ouvrent ces approches récentes a révélé ou rappelé aux historiens canadiens que cet intérêt pour l'histoire globale a inspiré toute une génération d'historiens européens. Il n'en fallait pas davantage pour que certains d'entre eux ambitionnent d'arracher aux enseignements de

* Une première version de ce mémoire a été présentée au colloque "Studying Human Behaviour in the Past - A Blueprint for Interdisciplinary Regional History" qui s'est tenu à l'Université de Western Ontario (London). Nous remerçions nos collègues à Carleton, à l'Université de Montréal et au Musée de l'Homme, ainsi que les participants au colloque pour leurs commentaires fort utiles sur différents aspects de notre communication. Leurs suggestions ont été fort appréciées même si toutes n'ont pu être retenues. Comme d'habitude, Rita Wallot nous a aidés de multiples façons.

1 S.Z. Klausner, ed., The Study of Total Societies (New York), 1967).

RHAF, vol. 33, no 3, décembre 1979 
l'École des Annales "la lumière blanche, unitaire" qui, selon Fernand Braudel, est indispensable à l'historien'2. Mais, ainsi que le notait Hanham en 1977, "Canadian historians have not yet been able to find thus far in the new history... satisfactorv approaches to many of their traditional sources of perplexity..."3. Et ce critique d'ajouter qu'on pouvait extraire beaucoup plus de l'École des Annales sans pour autant préciser quels pourraient être ces enseignements.

Dans les prochaines sections, nous énonçons, à propos de la connaissance historique, quelques réflexions qui serviront de fondement à notre perspective critique 4 sur l'utilisation de la problématique des Annales dans le contexte canadien, et de guide à notre tour d'horizon critique des thèmes et des approches inédites qui caractérisent l'historiographie récente sur le XIXe siècle canadien. Suivront ensuite une esquisse des contours de ce que pourrait être une problématique proprement canadienne et certaines directions de recherche qu'elle implique. Cette problématique, selon nous, doit parier sur la méso-analyse et déboucher sur la construction d'une méso-histoire du XIXe siècle canadien.

\section{LA CONNAISSANCE HISTORIQUE}

Pour notre propos, la définition du métier d'historien repose sur la réponse à deux questions fondamentales: que peut-on connaître du passé et comment accéder à cette connaissance?

2 F. Braudel, "Histoire et sociologie", G. Gurvitch, éd., Traité de sociologie (Paris, 1958), tome 1,96 .

3 H.J. Hanham, “Canadian History in the 1970's", The Canadian Historical Review [CHR], 58 (1977): 5.

4 Nous utilisons le mot "critique" dans l'acceptation qu'il a acquise pour l'École de sociologie critique de Francfort, i.e. non point comme pensée d'opposition mais comme réflexion sur les conditions inhérentes à toute approche qui en limitent l'efficacité et l'utilité. (P. Connerton, ed., Critical Sociology (Harmondsworth, 1976), 17-20). 


\section{a. L'objet}

L'histoire cherche à retracer ce qui s'est passé et pourquois. L'opération historique - "faire de l'histoire" - consiste donc à établir une série de rétrodictions et d'explications à propos d'un éventail plus ou moins large de réalités et d'événements passés. Pour fixer les idées, on peut départager ces réalités, comme Popper, en trois catégories: le monde 1 , celui des choses matérielles, le monde 2 ou le monde subjectif de l'esprit, de la connaissance et de la conscience, et le monde 3, celui des structures objectives produites par les hommes, intentionnellement ou non. Il s'agit donc pour l'histoire de décrire et de comprendre la dynamique du procès évolutif de cet univers complexe sans chercher à le réduire à un déterminisme moniste ou univoque à partir de l'un ou l'autre de ces mondes ${ }^{6}$.

On peut évidemment aborder l'analyse de cet univers en procès continu d'interaction et d'intercréation par le biais de l'examen de l'un ou l'autre de ces mondes. Cependant, comme le propose Popper, on a intérêt à attaquer d'abord le monde 3. (celui de l'organisation, des coutumes, lois, institutions humaines, droits, etc. $)^{7}$. Ce pari sur une approche institutionnelle/organisationnelle dans l'analyse du changement social n'est pas une réduction de cet univers complexe au monde 3, mais plutôt un choix stratégique. En effet, le monde 3 institue pour ainsi dire le procès socio-économique de façon à harmoniser les pressions en provenance des deux autres mondes - contraintes géo-techniques du monde 1 et valeurs, plans, projets et préférences émanant du monde 2 . En outre, comme la trame institutionnelle contient aussi des éléments ni voulus ni prévus, qu'elle ne dérive pas simplement des armistices entre faits de conscience et faits matériels mais surgit de l'interaction entre les

5 Il s'agit d'une définition assez conventionnelle reprise par beaucoup d'auteurs, v.g. N. Rescher, Essays in Philosophical Analysis (Pittsburg, 1969), chap. X; C. Hill, The Century of Revolution 1603-1714 (Bungay, 1969); E.H. Carr, What is History (London, 1965).

6 La ventilation des propositions historiques en rétrodictions et explications a été développée par Q. Gibson dans The Logic of Social Inquiry (London, 1960), chap. XV. En ce qui a trait à la distinction entre les mondes 1,2 et 3, K.R. Popper l'explicite tout au long de son livre Objective Knowledge (Oxford, 1972). Pour une présentation succincte des travaux de Popper, voir B. Magee, Popper (London, 1973). La "trinité" de Popper a certaines ressemblances avec celle suggérée par Boulding (K.E. Boulding, Ecodynamics $-A \mathrm{New}$ Theory of Societal Evolution (London, 1978), chap. X) - "things, people, organization"-.

7 K.R. Popper, op. cit., chap. VIII, 296ss. 
trois mondes, il semble qu'il soit préférable d'étudier la dymanique du procès évolutif en commençant par les caractéristiques objective du monde 3 pour ensuite déborder sur les deux autres mondes et les intégrer à l'analysę ${ }^{8}$.

Il devient évident que le genre d'explication historique à laquelle aboutit cette approche, ne peut s'exprimer à priori en termes de relations mécaniques univoques entre certaines variables ou certains sous-procès spécifiques (démographie, finance, idéologies, etc.), mais tout au plus en termes de relations cause-effet probables: des forces similaires tendent dans des circonstances semblables à produire des effets de même nature. Voilà qui engendre des explications stochastiques d'où sont bannis les déterminismes mécaniques et qui laissent leur place aux événements improbables, aux choix des hommes et aux conséquences nonvoulues et non-prévues 9 .

\section{b. La méthode}

Des désaccords importants opposent les praticiens en ce qui concerne la méthode à employer pour accéder à la connaissance historique. Un premier groupe estime que l'histoire peut et doit effectuer une reconstruction rationnelle du passé. Cette opération exige l'utilisation d'un corpus théorique, d'un outillage de conventions théoriques qui puissent guider cette reconstruction. Les partisans de cette position tiennent les historiens pour des scientifiques comme les autres, qui examinent les données, formulent des conjectures et hypothèses, en analysent les implications et confrontent ces implications à des données additionnelles. C'est pour

8 Il est évidemment possible d'amorcer l'enquête par le biais du monde 1 ou du monde 2; mais il semble que le risque est alors plus grand que ne s'accrédite un certain matérialisme ou un certain idéalisme dans les explications. Il faut de suite ajouter que cette proposition générale sur laquelle la majorité des historiens devraient pouvoir s'entendre porte sur le travail historique dans son ensemble. La division du travail historique a amené certains historiens à scruter un segment seulement de la trame institutionnelle du monde 3 ou à se concentrer sur certains rapports entre les entités du monde 3 et des mondes 1 ou 2 ou mêmeà enquêter sur certains aspects particuliers des mondes 1 ou 2. Ces travaux ont débouché sur des rétrodictions fort utiles ainsi que des explications partielles. La spécialisation qui entraîne certains à se confiner aux rétrodictions ou à certaines enquêtes partielles ou aux deux, résulte de toute division du travail. Notre intérêt porte sur le chantier historique et non sur la pratique de chaque ouvrier en particulier.

9 L'abandon du déterminisme mécanique cause-effet s'est accompli il y a déjà longtemps dans les sciences physiques. On peut se demander pourquoi l'étude des phénomènes culturels s'imposerait des paradigmes pré-heisenbergiens (M. Harris, Cannibals and Kings (New York, 1977), xi). Sur le rejet du "naïve determinism", voir T. Judt, "A Clown in Regal Purple: Social History and the Historians", History Workshop, 7 (1979): 70ss. 
façonner ces conjectures et hypothèses que l'historien doit avoir recours aux conventions théoriques. De là le nom de "conventionnalisme" que l'on a attaché à cette approche ${ }^{10}$.

Un second groupe insiste au contraire sur l'impossibilité de reconstruire rationnellement le passé: l'histoire ne serait pas une science mais un art; et cet art viserait à recréer des impressions $d u$ passé, une entreprise qui repose principalement sur l'intuition et une certaine forme d'induction à partir des données. L'intuition, le flair et un procès d'inférence à partir des faits identifiés par l'historien, telle serait la méthode. Voilà ce qu'on a nommé "inductivisme"11.

Ces vues extrêmes ne correspondent ni à des positions épistémologiques solides ni à la pratique quotidienne des historiens. Mais la virulence des attaques décochées de part et d'autre ne le laisse pas transparaître clairement. Voilà d'ailleurs pourquoi cette polarisation garde une valeur exemplaire, même si les deux extrêmes paraissent irrecevables tels quels. D'une part, il est clair que la connaissance n'est pas contenue dans les faits comme les partisans de l'induction et de l'art l'affirment, et que leur confiance aveugle dans la recréation impressionniste "pour le plaisir" à la Veyne ne saurait entraîner l'adhésion de la plupart des historiens. D'autre part, l'utilisation d'un corpus statique de conventions théoriques, nécessairement partielles et en usage à un moment donné, pour reconstruire l'expérience de socio-économies globales dans le temps long, ne peut que déboucher sur des images tronquées et des explications qui tournent court ${ }^{12}$.

En fait, les historiens ont souvent réussi à esquiver ces pièges en développant des modes de raisonnement qui cherchent la réconciliation efficace des deux tendances mentionnées plus haut. La pratique des historiens est devenue en général de plus en plus caractérisée par l'emploi de cadres conceptuels plus souples que ceux auxquels les "conventionnalistes" font appel, et par le recours

10 N. Rescher, op. cit., chap. IX. Frédéric Mauro soutient ce point de vue de façon particulièrement éloquente: “... l'histoire est discipline théorique donc scientifique... l'histoire n'est donc pas de nature différente de celle des autres sciences sociales". (Des produits et des hommes (Paris, 1972), 29-31). On trouvera de nombreux arguments en faveur de cette position également dans C.C. Hempel, "Explanation in Science and History", W.H. Dray, ed., Philosophical Analysis and History (New York, 1966); G. Myrdal, "How Scientific Are the Social Sciences", Economies et sociétés, 6 (1972): 1473-1496.

11 P. Veyne, Comment on écrit l'histoire: essai d'épistémologie (Paris, 1971). Voir aussi I. Berlin, "The Concept of Scientific History", W.H. Dray, ed., op. cit.

12 L.A. Boland, "Time in Economics vs Economics in Time", Revue canadienne d'économique, 11 (1978): 240-262. 
à des procédés de vérification d'hypothèse très flexibles qui tiennent compte du fait que le cadre conceptuel est lui-même en évolution.

Au centre de la méthode historique se situe la transduction. Cette opération intellectuelle, ainsi appelée par Henri Lefebvre, "diffère de l'induction, de la déduction classique et aussi de la construction de 'modèles', de la simulation, du simple énoncé des hypothèses. La transduction élabore et construit un objet théorique, un objet possible et cela à partir d'informations portant sur la réalité ainsi que d'une problématique posée par cette réalité. La transduction suppose un feedback incessant entre le cadre conceptuel utilisé et les observations empiriques"13.

Ainsi, la notion d'un cadre conceptuel donné et fermement défini à un moment du temps est remplacée par la notion d'une succession de conceptualisations qu'il faut apprécier comme faisant partie intégrante d'un programme de recherche en marche qui, pour ainsi dire, soude les versions successives. On voit que le raccord entre le cadre conceptuel et le réel veut bien davantage contribuer à améliorer la conceptualisation qu'à la réfuter. Le cadre conceptuel est en quelque sorte immunisé contre la réfutation brutale et la "cruauté méthodologique" est bannie: au lieu d'accepter qu'une exception permette de rejeter la règle, on ne veut renvoyer que les règles qui déclarent impossibles des états de faits observables ${ }^{14}$.

$\mathrm{Au}$ point de départ du procès de transduction, de cette séquence cumulative de corrections à mesure que le cadre conceptuel enregistre le feedback incessant du réel, se trouve la problématique - un ensemble de problèmes ou de questions ou les deux

13 H. Lefebvre, "Pour un nouvel urbanisme", Revue française de sociologie, juilletseptembre 1961; aussi idem, Le droit à la ville (Paris, 1968), 121.

14 Pour une discussion des différences importantes entre les mécanismes un peu naïfs de vérification d'hypothèse et la méthodologie plus souple dite des programmes de recherche, on consultera avec profit I. Lakatos, "Falsification and the Methodology of Scientific Programmes", I. Lakatos et A. Musgrave, ed., Criticism and the Growth of Knowledge (Cambridge, 1970), spécialement la section 2. Cette méthodologie "évolutionnaire" asseoit ses fondements sur le postulat que la connaissance est cumulative et que l'on ne saurait isoler une proposition simple aux fins de test du programme de recherche dont elle fait partie. Voilà qui impose des normes de tolérance méthodologique: il arrive souvent que nous sachions bien que certaines propositions théoriques sont irrecevables sans que pour autant le programme de recherche (dont ces propositions sont partie intégrante) cesse d'avoir une grande utilité pour guider l'enquête. Et, de commenter Lakatos, "to give a stern 'refutable interpretation' to a fledging version of a programme is dangerous methodological cruelty" (p. 151). 
qui, même formulés de façon plus ou moins précise, sont considérés par le chercheur comme des dimensions fondamentales pour la compréhension de l'évolution du procès socio-économique. L'ensemble de questions/problèmes constituant la problématique forme un noeud complexe dont la texture peut être poreuse mais qui a une intégrité propre, une dérive particulière ${ }^{15}$. Même quand la problématique semble revêtir bien davantage un pouvoir d'évocation qu'une capacité à guider effectivement l'enquête, il n'en reste pas moins qu'elle peut fournir des indications utiles, suggérer des questions et des problèmes additionnels et, ce faisant, être à l'origine de nouvelles directions pour l'enquête, de déplacements du centre de gravité du programme de recherche.

\section{c. Coda}

L'historien tente de décrire et d'expliquer la dynamique du procès évolutif des mondes 1,2 et 3 en interaction. Nous avons argué qu'il y avait certains avantages à procéder à partir du monde 3 à l'aide d'une problématique et d'une opération intellectuelle nommée transduction. Mais au point de départ de cette connaissance historique se trouve le choix de la problématique. Choix crucial puisque la problématique tend à engendrer ses propres questions et à guider l'enquête via le procès de transduction dans certaines directions. Or, il existe évidemment des problématiques concurrentes, et il s'agit pour les chercheurs de jauger le potentiel heuristique particulier de chacune des problématiques disponibles, c'est-à-dire leur capacité relative à indiquer au chercheur quelles voies éviter et quelles avenues choisir ${ }^{16}$.

15 La notion de problématique a été utilisée par G. Bachelard, Le rationalisme appliqué (Paris, 1949), 51ss. Il s'agit de schèmes d'interprétation qui peuvent varier considérablement en étendue, en spécificité et en profondeur (voir $\mathrm{H}$. Leibenstein, Beyond Economic Man (Cambridge, 1976), chap. II). L'utilisation de problématiques est fort répandue en histoire. On peut mentionner dans le contexte canadien l'approche en termes des produits générateurs popularisée par Harold Innis. Une autre à vocation plus vaste et dont traitera la prochaine section est l'approche dite de l'École des Annales. Enfin l'on peut mentionner celle qui met l'accent sur les droits de propriété (A.A. Alchian \& H. Demsetz, "The Property Rights Paradigm", Journal of Economic History, 1973) ou les différentes problématiques à saveur marxienne ou marxiste (voir P. Vilar, "Histoire marxiste, histoire en contruction", J. Le Goff et P. Nora, éd., Faire de l'histoire (3 vol., Paris, 1974), tome 1: 169209). Dans chaque cas, la problématique constitue en quelque sorte une vision du monde qui sert de point de départ et de principe souple d'organisation de l'enquête.

16 Sur la notion de potentiel heuristique ("heuristic power") d'une problématique, voir I. Lakatos, op. cit., section 3(a), 3(b) et 3(d) 4 en particulier. 
Le choix de la meilleure problématique consistera à opter pour l'approche qui assure cumulativement la meilleure compréhension et l'explication la plus satisfaisante de la dynamique des trois mondes en interaction.

\section{L'ÉCOLE DES ANNALES}

La considération de l'École des Annales présente pour nous un double intérêt. D'une part, on l'a vu, certains ont suggéré que l'approche des Annales pourrait avoir valeur exemplaire pour l'historiographie canadienne: elle serait celle qui peut fournir la problématique ayant le plus grand pouvoir heuristique pour les historiens canadiens et elle mérite donc une attention spéciale en tant que telle. D'autre part, il s'agit d'une approche, d'un programme de recherche ambitieux et déjà assez ancien pour qu'il nous serve de terrain d'expérimentation dans l'utilisation des catégories développées dans la dernière section. En particulier, nous voudrions mesurer en quelques sorte les déplacements du cadre conceptuel des Annales dans le temps.

Pour prometteur que pouvait paraître cet excursus, notamment parce que l'École des Annales a toujours insisté sur le fait qu'ill n'y a pas d'histoire sans problématique explicitée..."17, l'enquête s'est avérée plus difficile que prévu: en effet, cette "École" s'est montrée peu explicite dans la présentation de sa problématique et de sa méthodologie. Louis Michel pouvait écrire à son propos en 1974: "Ni théorie, ni système, ni méthodologie spécifique, elle est avant tout une pratique caractérisée par des attitudes, des choix, des ambitions." 18 Or voilà justement de quoi est constituée une problématique. Cette problématique a dérivé dans le temps: nous avons donc cherché à la pister à travers les trois générations de l'École des Annales.

\section{a. Génération I}

Lucien Febvre et Marc Bloch incarnent la première génération de l'École des Annales. Première génération qui a produit une nouvelle approche et quelques nouveaux concepts, et a cerné de nouveaux objets d'étude historique dans ses efforts de recons-

17 P. Chaunu, "L'histoire sérielle: bilan et perspectives", Revue roumaine d'histoire, 9 (1970): 473

18 L. Michel, "Le territoire de l'historien d'Emmanuel Le Roy Ladurie", RHAF, 28, 1 (juin 1974): 96. 
truction/recréation du passé. Sous l'influence de spécialistes français des sciences sociales du début du XXe siècle, tels Vidal de la Blache en géographie et Durkheim en sociologie, Febvre et Bloch déployèrent des efforts remarquables pour faire de l'histoire "le carrefour des sciences humaines", pour composer ces dimensions, éclairées par les spécialistes des sciences humaines, en une prise sur la totalité.

L'approche de ces deux pionniers insistait sur l'importance du temps long et était fermement comparatiste. Il fallait, disaient-ils, ne pas s'arrêter aux simples événements individuels: on ne pourrait prendre le pouls du procès évolutif qu'en situant les événements particuliers dans leurs coordonnées synchroniques et diachroniques. En d'autres mots, pour comprendre le procès, il fallait le lire en quelque sorte horizontalement et verticalement, un peu comme une partition musicale.

Febvre et Bloch perçoivent également la dualité des "faits matériels" et des "faits moraux", qui ont bien des affinités avec les mondes 1 et 2 évoqués plus haut. Mentalités, outillage mental, genre de vie: autant de catégories parmi d'autres qu'ils développèrent, affinèrent et adaptèrent dans leur effort pour explorer les interactions entre ces mondes et la façon qu'ils avaient de se réfléter dans le monde 3 des institutions. Mais cet effort gigantesque pour reconstruire l'infrastructure de la pensée de certaines époques ou "une archéologie du quotidien" (Duby), ainsi que leurs explorations du monde 3 , s'effectuèrent en général via des méthodes presque exclusivement inductives.

On peut imputer cette stratégie inductive à certains postulats irréalistes quant au pouvoir de "révélation" des documents euxmêmes et des données brutes. Voilà qui ressort clairement dans le livre de Bloch, Apologie pour l'Histoire. Cependant, cette conséquence résulte aussi, selon les mots mêmes de Fernand Braudel, d'une "difficulty in stating problems, fixing benchmarks and drafting a methodology", difficulté qui a affligé la première génération de l'École des Annales. Cette position méthodologique devait réduire le pouvoir heuristique de la problématique des Annales et entraîner des effets d'écho fâcheux sur les générations ultérieures. 
Il reste qu'un programme de recherche débute avec Febvre et Bloch, qu'une stratégie ambitieuse et audacieuse est esquissée: l'esprit des Annales est né ${ }^{19}$.

\section{b. Génération II}

La seconde génération, c'est, pour nous comme pour Peter Burke, celle de Braudel, Chaunu, Goubert et Le Roy Ladurie, encore qu'il ne s'agisse que des chefs de file ${ }^{20}$. Comme groupe, ils ont enrichi et élargi la problématique d'une multitude de façons à travers des études à première vue un peu hétérogènes.

Deux traits marquants se dégagent des travaux de cette seconde génération. Premièrement, les comparaisons deviennent plus ambitieuses et le temps long, encore plus long. La première oeuvre majeure de Braudel portait sur la Méditerranée, celle de Chaunu, sur le système atlantique. Les travaux plus récents de Braudel traitent du "système mondial" et couvrent la période 14001800 alors que l'étude du climat entreprise par Le Roy Ladurie commence en l'an mil21. Ce cadre plus large, cette "pesée globale" pour reprendre l'expression de Chaunu quand il parle des travaux

19 Ce portrait de l'École des Annales demeure évidemment un peu impressionniste. Notre propos n'est pas de faire l'histoire de l'École, mais seulement de découper certaines arêtes de la problématique qui marquent des moments différents dans l'évolution du groupe. On trouvera des précisions sur l'esprit des Annales dans l'introduction que Georges Duby a écrite pour la réédition récente du livre célèbre de Marc Bloch, Apologie pour l'histoire (Paris, 1974). Duby y analyse brièvement le riche contenu du livre dans le contexte du chantier ouvert par Febvre et Bloch. Pour les suites de ce défrichement, voir J. Le Goff et P. Nora, éd., Faire de l'histoire. Lire aussi les commentaires opportuns de Braudel sur Febvre dans The International Encyclopedia of Social Sciences, 1968.

20 P. Burke, ed., Economy and Society in Early Modern Europe (New York, 1977), introduction. Les avis diffèrent considérablement quant à l'identification des générations. Ainsi, Louis Michel considère que Febvre, Braudel et Le Roy Ladurie représentent trois générations successives des Annales ("Le territoire de l'historien [...]"). À notre avis, bien que Le Roy Ladurie soit plus jeune et ait remplacé Braudel au Collège de France, ses concepts et sa méthodologie le rattachent à la génération de Braudel. La référence de Michel à la quantification comme caractéristique principale de la "troisième" génération sous-estime l'importance des travaux quantitatifs de Braudel (notamment avec Spooner). Toutefois, si le schème de démarcation proposé par Michel ne nous agrée pas, il reste que la position de Le Roy Ladurie n'est pas sans une certaine ambiguïté. On pourrait parler de deux Le Roy Ladurie: en effet, ainsi que nous le verrons plus loin (v.g. note 28), certains de ses travaux ont une saveur qui déborde sur la troisième génération.

21 Voir F. Braudel, La Méditerranée et le monde méditerranéen au temps de Philippe II (2 vol., Paris, 1949 et 1967) (édition revue); idem, Civilisation matérielle et capitalisme, tome 1 (Paris, 1967) - deux ou trois autres volumes sont annoncés pour un proche avenir; P. Chaunu, Séville et l'Atlantique de 1504 à 1650 (8 vol., Paris, 1955-1959); E. Le Roy Ladurie, Histoire du climat depuis l'an 1000 (Paris, 1966); idem et J. Goy, éd., Les fluctuations du produit de la dîme (Paris, 1972) - un second volume sur les dîmes doit paraître en 1980 . 
de Braudel ${ }^{22}$, confère aussi une valence plus grande aux facteurs géo-économiques (dans la foulée de l'économisme prudent du Bloch de la dernière période) et une plus grande importance à la quantification (sous l'influence notamment de Simiand et Labrousse). D'ailleurs, ces derniers, de même que Henri Sée et Georges Lefebvre, ont marqué l'historiographie française autant sinon davantage que l'École des Annales.

Deuxièmement, la seconde génération a confirmé le projet d'une histoire totale lancé par Febvre et Bloch. Chaunu écrit de Braudel que sa pensée est "une pensée de la totalité... l'histoire totale, synthèse la plus humaine de toutes les sciences de l'homme"23. Evidemment, tous les membres de la seconde génération n'ont pu relever le défi avec la même élégance et continuer ce projet d'histoire totale avec le même succès; mais des virtuoses comme Braudel et Le Roy Ladurie ont réussi à tisser des structures polyphoniques majestueuses dans leurs partitions. On peut référer aux travaux réussis de Braudel et Chaunu sur un mode macroscopique et à ceux de Le Roy Ladurie et Goubert sur un mode plus microscopique ${ }^{24}$.

Alors que pour la première génération, les forces privilégiant le temps long et les comparaisons étaient plus ou moins équilibrées par un accent tout aussi important sur le projet d'histoire totale, dans la seconde génération, la première série de forces prend le pas sur la seconde. Certains travaux de la seconde génération semblent même renoncer à l'histoire totale ${ }^{25}$. Voilà qui devait conduire à une

22 P. Chaunu, "La pesée globale en histoire", Cahiers Vilfredo Pareto, 15 (1968): 135-164, reproduit dans idem, Histoire quantitative - histoire sérielle (Paris, 1978), 35-54. "Cette conception... postule que le destin de l'homme découle des constantes de la géographie et du climat et de la lente évolution des conjonctures économiques..." (H.L. Wesseling, "La transformation du 'world system' à la fin du dix-neuvième siècle et l'empire colonial néerlandais", Europa, 1 (1977): 37).

23 P. Chaunu, "La pesée globale en histoire”, 163-164.

24 Il ne faudrait pas oublier que cet effort de la seconde génération pour parvenir à une histoire totale a entraîné une certaine division du travail historique. Des dimensions nouvelles des mondes 1, 2 et 3 ont été scrutées en grand détail par certains historiens dans leurs synthèses "régionales". Parmi les exemples bien connus, mentionnons E. Le Roy Ladurie, Les paysans du Languedoc (2 vol., Paris, 1966); idem, Montaillou village occitan (Paris, 1975); P. Goubert, Beauvais et la Beauvaisie de 1600 à 1730 (2 vol., Paris, 1960); R. Mandrou, De la culture populaire au XVIIe et XVIIIe siècle, la bibliothèque bleue de Troyes (Paris, 1964). Certains travaux récents de Ladurie se déplacent vers des comparaisons internationales et une échelle plus macroscopique (climat, dîmes, production agricole, etc.).

${ }_{25}$ Pensons aux études de longue durée sur l'évolution du produit de la dîme, le mouvement des loyers, l'évolution de la monnaie, etc. (voir certains exemples dans E. Le Roy Ladurie, Le territoire de l'historien, tome 1 (Paris, 1973). 
certaine primauté du diachronique, de la longue durée sur la complexité de la structure synchronique dans les travaux de la seconde génération; à l'émergence d'une certaine promotion, d'une certaine primauté de la série chronologique comme forme de réduction privilégiée et comme confirmation dans une forme renouvelée de l'inductivisme de l'école des Annales $^{26}$. Chaunu est peut-être celui qui a poussé le plus loin dans cette direction ou tout au moins celui dont le discours est le plus clair sur ces questions. N'a-t-il pas lancé sous diverses formes: "Une fois constituée, la série porte en elle ses propres raisons!" 27

\section{c. Génération III}

Depuis une quinzaine d'années s'est affirmé de plus en plus le besoin de réintroduire fermement l'importance de la synchronie, d'échapper au réductionnisme de la longue durée: une troisième génération de l'École des Annales est en train de prendre. Celle-ci se cherche encore un nom. Ses membres participent à la fois du nouveau et de l'ancien, car si l'on sent bien qu'il y a eu évolution dans la problématique de certains représentants de la seconde

26 Il n'est pas sûr qu'il s'agisse d'un choix délibéré, mais la seconde génération partage avec la première un certain manque de goût pour la réflexion épistémologique et méthodologique. Même chez Braudel, le chef de file de la seconde génération et celui qui a le plus écrit sur les problèmes de méthode, on retrouve le goût de cette "totalisation inductive" qui s'enracine dans l'accumulation des données constituées en séries. Son insistance sur la longue durée le conduit même à télescoper ou à aplatir et la structure des temps sociaux et la structure de la réalité sociale en une seule dimension: pour lui, la dominance de la longue durée est celle des forces géo-économiques, car "la longue durée, c'est l'infrastructure". Fourquet met en lumière un aspect intéressant de cette réduction chez Braudel: il montre comment celui-ci met l'accent sur la dimension diachronique (d'une façon très voisine de "l'histoire sérielle") et présume qu'il y a un lien univoque entre des types de temps sociaux et de durée, d'une part, et les types ou les "paliers" de la structure de la réalité socio-matérielle, d'autre part. Certains aspects de cette tendance à la réduction transparaisent également dans la controverse entre Braudel et Gurvith. Voir F. Fourquet, L'idéal historique, 86-91 et G. Gurvitch, La multiplicité des temps sociaux (Paris, 1958).

${ }_{27}$ P. Chaunu, "L'histoire sérielle", RHAF, 21,1 (septembre 1967): 182. Chaunu est le plus éminent propagandiste de l'histoire sérielle. Celle-ci, à ses yeux, accomplit "une mission fédératrice dans la grande famille des sciences humaines". Dans la préface à son Histoire quantitative - histoire sérielle, il affirme sous le couvert d'une fausse interrogation: "Y a-t-il encore aujourd'hui une forme d'histoire qui ne soit pas... histoire quantitative ou histoire sérielle?" (p. 5). Braudel avait pourtant critiqué (dans un compte rendu par ailleurs favorable) cette sorte de foi en concluant sur le "besoin de sortir des courbes" (F. Braudel, "Pour une histoire sérielle: Séville et l'Atlantique (1504-1650)", Annales E.S.C., 18 (1963): 541-553). Pour une critique polémique de cet inductivisme, voir T. Judt, "A Clown in Regal Purple: Social History and the Historians". 
génération, on doit constater aussi l'ajout de ceux qui, au dire de Le Goff et Nora, "les continuent dans l'innovation", v.g. les Burguière, Goy, Revel, etc. ${ }^{28}$

Ces tâtonnements ont focalisé l'attention sur le besoin de redéfinir ou tout au moins de réorienter "l'opération historique". Pour Michel de Certeau, l'un des principaux protagonistes dans les débats actuels, la nouvelle approche ne peut se fonder sur l'accumulation des données et sur un effort de "totalisation inductive" à la manière de la seconde génération. Elle doit plutôt se concentrer sur la trame institutionnelle, sur "l'hétérogène, les différences, les écarts"; elle doit opérer avec un "outillage d'emprunt" provenant des autres sciences sociales à partir d'“une cohérence initiale", puis procéder "sur le mode de l'expérimentation critique" 29 . Ce n'est pas trop dire que cette troisième génération semble avoir reconnu l'importance stratégique du monde 3 (institutions/organisations) comme porte d'entrée et l'utilité du couple problématique/transduction comme méthode.

Le nouvel accent sur la synchronie a poussé la troisième génération à modifier le programme de recherche: "L'historien... ne vise plus le paradis d'une histoire globale" 30 , il aspire plutôt à une histoire qualifiée de "générale". Au lieu de vouloir embrasser une cohérence globale difficile à appréhender et peut-être absente, la troisième génération a privilégié l'hétérogénéité du procès socio-

28 On sent la dérive dans cette direction quand Chaunu parle du recours de l'histoire à "la pensée structurelle, structuraliste si l'on veut", ou quand Le Roy Ladurie évoque "l'histoire événementialo-structurale" dans sa discussion du soulèvement chouan comme reflet pour ainsi dire des structures économiques et mentales. Voir P. Chaunu, "La pesée globale en histoire", 163; E. Le Roy Ladurie, Le territoire de l'historien, 1: 169-186. Quelques leaders de ce renouvellement sont mentionnés plus loin.

${ }_{29}$ M. de Certeau, "L'opération historique", J. Le Goff et P. Nora, éd., Faire de l'histoire. Ce chapitre constitue un apport important aux réflexions des historiens sur leur pratique et se rapproche des notions de problématique/transduction esquissées au début de cette section. De Certeau cite même Popper. Il réfère à une étude méthodologique de Braudel lorsqu'il insiste sur les facteurs structuraux, les différences et l'hétérogénéité ( $\mathrm{F}$. Braudel, Écrits sur l'histoire (Paris, 1969), particulièrement les pages 292 ss). On peut contester notre lecture de cette troisième génération. Toutefois, dans la présentation du livre dont le chapitre de Certeau est le premier texte, les éditeurs font remarquer que "si l'on retrouvera chez les auteurs et dans l'esprit de l'ouvrage la marque souvent de la prétendue école des "Annales", c'est que l'histoire nouvelle doit beaucoup à Marc Bloch, à Lucien Febvre, à Fernand Braudel et à ceux qui les continuent dans l'innovation". (Op. cit., ix).

30 P. de Certeau, op. cit., 27. 
économique, le caractère essentiel de la dimension verticale dans la "partition", la nécessité de construire non pas une série mais un tableau ${ }^{31}$.

On peut donc conclure que cette troisième génération élabore des histoires à la fois plus précises et plus ambitieuses. Les travaux de Michel Foucault sur la clinique, la prison, la sexualité, et ses conjectures sur les corrélations plausibles, les dominantes, les isomorphismes et les analogies entre les dimensions du monde 3, illustrent bien les potentialités de cette histoire générale ${ }^{32}$.

\section{d. En guise de conclusion}

Il ne fait pas de doute que l'on peut identifier dans l'expérience des Annales les contours d'une problématique en marche. On peut aussi repérer les déplacements de son centre de gravité dans le temps. Il n'est pas inintéressant de constater qu'à la troisième génération, la problématique a évolué nettement vers un objet et une méthode qui rejoignent assez bien les lignes de force auxquelles nous en étions arrivés à la fin de la section sur la connaissance historique.

31 “Le problème qui s'ouvre alors - et qui définit la tâche de l'histoire générale c'est de déterminer quelle forme de relation peut être légitimement décrite entre ces différentes séries; quel système vertical elles sont susceptibles de former; quel est, des unes aux autres, le jeu des corrélations et des dominantes; de quel effet peuvent être les décalages, les temporalités différentes, les diverses rémanences; dans quels ensembles distincts certains éléments peuvent figurer simultanément; bref, non seulement quelles séries, mais quelles "séries de séries" - ou en d'autres termes, quels "tableaux" il est possible de constituer. Une description globale resserre tous les phénomènes autour d'un centre unique - principe, signification, esprit, vision du monde, forme d'ensemble; une histoire générale déploierait au contraire l'espace d'une dispersion". (M. Foucault, L'archéologie du savoir (Paris, 1969), 1819).

32 Pour une esquisse de la tradition intellectuelle française où s'insère le mouvement structuraliste et une appréciation de l'impact de l'épistémologie structuraliste et de la nouvelle histoire culturelle à la Foucault, voir E. Morot-Sir, La pensée française d'aujourd'hui (Paris, 1971). Un survol des travaux de Foucault a été publié sous forme de dossier dans le Magazine littéraire (juin 1975). Les structuralistes mettent en relief la dimension synchronique (parfois d'une façon qui semble exclure le temps et la durée, mais ce biais n'est pas inhérent à l'approche) et détectent les isomorphismes entre les différents paliers ou niveaux. En un sens, leur insistance sur les structures n'est qu'un rappel qu'on ne peut analyser de façon significative, un système de relations (une langue, une socio-économie, un système de transmission des patrimoines, etc.), i.e. les parties d'une totalité, sans référence à la totalité. Pour une étude critique du concept de structure, voir R. Boudon, $\dot{A}$ quoi sert la notion de structure? (Paris, 1968). Pour un exemple d'isomorphisme audacieux présenté récemment, voir J. Attali, Bruits (Paris, 1977), où l'auteur prétend “juger une société sur ses bruits"(p. 7). On retrouve, derrière beaucoup de travaux et enquêtes de l'École des Hautes Études en Sciences sociales sur les patrimoines, les stratégies matrimoniales, etc., des efforts pour découvrir le code qui donnerait la clé de la structure du monde 3 et de son évolution. Pour une approche similaire dans l'histoire de la culture matérielle, voir H. Glassie, Folk Housing in Middle Virginia. A Structural Analysis of Historical Artifacts (Knoxville, 1975). 
Cependant, si l'étude de la problématique des Annales peut servir à mieux comprendre les démarches suggérées dans la section 2 , il ne s'ensuit pas que cette problématique recèle des enseignements importants ou du moins immédiatement utilisables pour les historiens du XIXe siècle canadien. On peut encore moins la brandir comme un talisman ("a charm supposed to benefit or protect the possessor, especially by exerting magical or occult influence" Funk et Wagnalls) pour justifier tout programme de recherche. D'autant plus qu'à notre avis, les emprunts qu'on a multipliés à l'École des Annales au Canada semblent s'adresser avant tout aux deux premières générations plutôt qu'à la troisième ${ }^{33}$.

Les Annales - et d'autres influences comme celles de Labrousse et de Georges Lefebvre - ont évidemment incité les Canadiens à fouiller de nouvelles sources (archives notariales, registres d'État civil, comptabilités des communautés religieuses, etc.). Mais le manque de netteté de la problématique sous-jacente et le manque de goût pour une réflexion sereine sur les méthodes utilisées ont empêché qu'il n'en sorte l'épure d'un programme de recherche sur le XIXe siècle canadien. D'autre part, l'inductivisme des Annales était souvent absorbé sans que l'emprunteur ne s'en rende bien compte. Voilà qui devait bloquer l'analyse à un niveau trop bas. Ceux qui souhaitent le plus un impact décisif des Annales semblent réduits à émettre le voeu qu'arrive le moment où "there will at last emerge a Braudel" au Canada ${ }^{34}$. Le pouvoir heuristique d'une problématique est assez réduit s'il faut se satisfaire d'espérer "en attendant Godot".

Bien plus, on peut se demander si l'importation sans adaptation ni innovation de cette problématique floue, si l'écho des deux premières générations des Annales sur l'histoire du XIXe siècle canadien, n'a pas jusqu'à un certain point desservi les disciples trop empressés. En effet, les travaux de ces générations ont porté presque exclusivement sur la période d'avant le XIXe siècle et sur des milieux relativement fermés et stables, porteurs d'une lente mouvance. Une transplantation sans précaution de cette approche dans

33 Voilà qui est très clair à l'examen des travaux les plus ambitieux inspirés par l'École des Annales au Canada: v.g. ceux de F. Ouellet, L'Histoire économique et sociale du Québec, 1760-1850 (Montréal, 1966); idem, Le Bas-Canada, 1791-1840. Changements structuraux et crise (Ottawa, 1976), l'approche résolument "annaliste" du groupe Landon, etc. Voir H.J. Hanham, op. cit., ainsi que le texte d'Alfred Dubuc sur l'influence des Annales au Canada dans la présente livraison de la RHAF.

34 H.J. Hanham, op. cit., 13. 
le XIXe siècle canadien, dans une colonie du nouveau monde caractérisée par une grande turbulence des populations et des institutions, par un développement fort différent de celui de sociétés qui ont atteint la stabilité de croisière du monde vieux et plein, par une transition relativement rapide vers le capitalisme industriel, peut fort bien avoir engendré des analyses qui portent à faux ${ }^{35}$.

\section{LE XIXe SIẼCLE CANADIEN: HISTORIOGRAPHIE DE LA DERNIÊRE DÉCENNIE}

Les historiens intéressés au XIXe siècle canadien ne sont pas tombés d'accord sur une problématique spécifique qui pourrait baliser leurs travaux. Cependant, leurs pratiques variées, déterminées souvent par les contraintes qu'imposent les sources de données disponibles et leur participation aux divers réseaux collégiaux de communication européens et nord-américains, ont acquis certains traits distinctifs. Ceux-ci se sont précisés suffisamment au cours de la dernière décennie pour qu'il soit possible de parler d'une problématique latente, d'une problématique en train de prendre dans l'historiographie portant sur le XIXe siècle canadien.

\section{a. Une problématique en train de prendre}

Dans la conclusion de son étude sur le discours des historiens canadiens-anglais des deux premiers tiers du XXe siècle, Carl Berger constate qu'ils ont tous été "nationalist of various hues, and sometimes their judgments about what was central to the past and what was peripheral arose as much from divergent conceptions of nationality as from disagreements about interpretations of the same evidence" 36 . Cette généralisation prudente s'applique également à l'historiographie canadienne-française de la même période.

35 Nous avons souligné ces difficultés à quelques occasions: G. Paquet et J.-P. Wallot, "The Agricultural Crisis in Lower Canada, 1802-1812: mise au point", CHR, 56 (1975), 146 ss; idem, "Rentes foncières, dîmes et revenus paysans: le cas canadien", communication au colloque international d'histoire rurale, Paris, juillet 1977, section b (à paraître). H.L. Wesseling note lui aussi ce caractère pré-XIXe siècle et bien qu'il loue cette "histoire moderne renouvelée", il craint que l'on commette des anachronismes en voulant appliquer l'approche des Annales aux sociétés des XIXe et XXe siècles. (op. cit., 37-39).

36 C. Berger, The Writing of Canadian History (Toronto, 1976), 259. "[This is] as close as Berger comes to asserting an overt thesis" (A.B. McKillop, "Carl Berger and Canada's Past Masters", Queen's Quarterly, 84 (1977): 623). 
Un tel point de vue ne rend pas justice aux historiens régionaux et locaux comme Harvey, MacNutt, Talman, Kerr, Ormsby, etc. "whose solid local and regional studies provided the basis for both specific detail and substantive generalizations" ${ }^{37}$. Il n'en reste pas moins vrai que le gros de l'historiographie canadienne a mis "undue emphasis on national history and unity", ignorant en pratique sinon en théorie les régions, les cultures, les ethnies, c'est-àdire l'hétérogène, les différences, les écarts qui définissent la trame institutionnelle du monde 3 (institutions/organisations) canadien.

Ce qui émerge des dix dernières années d'historiographie sur le XIXe siècle canadien, c'est le postulat que la diversité constitue la spécificité du Canada et, avec ce postulat, la conviction que si on veut comprendre l'histoire du Canada, il faut développer une pluralité d'histoires correspondant à la pluralité des expériences séparées mais non indépendantes qui s'entrecroisent pour former le lacis de l'expérience canadienne.

Cet intérêt nouveau des historiens canadiens correspondait à une certaine "impatience" à l'égard des problématiques macroscopiques qui avaient pétri l'historiographie canadienne dans les deux premiers tiers du XXe siècle ${ }^{38}$. Il devait s'ensuivre une véritable explosion de travaux de toutes sortes dans un grand nombre de directions: études s'attachant à l'examen de couches sociales, de régions, de sections, de groupes ethniques et culturels, de tranches

37 A.B. McKillop, op. cit., 624.

38 L'interprétation "laurentienne" de l'histoire canadienne, issue notamment de l'influence conjuguée de la thèse de Innis sur le produit générateur et de celle de Creighton sur la construction du Canada à partir de l'axe du Saint-Laurent, a suscité de nombreuses réactions depuis deux décennies. Citons Careless, qui a plutôt mis en valeur le rôle de centres métropolitains et régionaux drainant les ressources de l'arrière-pays et étant en communication avec d'autres métropoles ("métropolitanisme"), l'émergence d"identités limitées" à travers lesquelles les Canadiens des différentes régions ont interprété leur "nation" comme tout (J.M.S. Careless, "Limited Identities in Canada", CHR, 40, 1969; C. Berger, The Writing of Canadian History); W.L. Morton ("Clio in Canada: The Interpretation of Canadian History", University of Toronto Quarterly, 1946; "Some Thoughts on Understanding Canadian History", Acadiensis, 2, printemps 1973) et Lower qui ont valorisé les régions. Pour un bref survol de ces thèses, voir Pat Roy, "The Most Famous Textbooks", CHR, 57 (1976): 180-188. Ce n'est pas un hasard si un manuel récent s'intitule Canada: unité et diversité (P. Cornell et al. (Toronto, 1967). 
de la socio-économie canadienne. Travail de déblayage nécessaire mais aussi un peu chaotique; coups de sonde que n'est pas venue encadrer une perspective intégratrice.

À cause de la pauvreté du fichier documentaire, on a conféré une importance indue à la simple accumulation de données considérables dont souvent on ne savait que faire. De plus, la timidité (ou la prudence) naturelle des historiens a exclu les généralisations audacieuses en l'absence de monographies suffisamment solides et abondantes pour les étayer. Plus encore, ces lacunes documentaires ont excusé les historiens de reporter à plus tard les débats de fond sur la meilleure façon de découper la socio-économie canadienne, lors même qu'il était acquis qu'il ne saurait être question de considérer une seule réalité nationale, mais plutôt une multiplicité, une diversité d'expériences.

Ce problème a produit d'importants effets de retombée sur le travail historique: se sont vite accrédités des découpages traditionnels (régionaux ou sectoriels ou socio-professionnels, etc.) qui ont ambitionné de départager le procès socio-économique avec la netteté et le simplisme de ces schèmes classificatoires d'antan qui classaient les animaux en groupes selon le nombre de pattes ${ }^{39}$.

On ne saurait nier l'existence de premières tentatives pour dégager des partitions de la socio-économie canadienne qui soient plus utiles. Les travaux de Careless sur le "métropolitanism" - cum - "limited identities", la variété des schèmes centrepériphérie ou de développement du sous-développement qui ont tenté de construire une image de la structure d'inégalité qui caractérise l'architecture socio-économique du pays, etc., peuvent être considérés comme autant de premières tentatives pour intégrer les

39 On ne connaît bien que ce que l'on reconstruit. Le problème du découpage dans les sciences sociales ne diffère pas tellement qu'il s'agisse du cas d'une réalité socio-économique complexe ou du cas plus simple d'un départage géographique d'une région. Pour une étude du problème du découpage en général, voir G.G. Granger, Pensée formelle et science de l'homme (Paris, 1960), chap. IV. Pour une application au découpage de régions en géographie, voir $P$. Haggett, Locational Analysis in Human Geography (London, 1965), chap. IX. 
travaux riches, hétérogènes et complémentaires de la nouvelle génération des historiens qui ont étudié le XIXe siècle ${ }^{40}$. Nous verrons dans la prochaine section que ces travaux ont bien imparfaitement réussi à guider et à canaliser la recherche; mais ils constituaient les premières étapes dans l'exploration d'une problématique et d'un programme de recherches pariant sur l'unité dans la diversité.

\section{b. Le bouillonnement récent de l'historiographie}

En évitant le télescopage des expériences des régions et des groupes divers en un seul procès "national" et selon un seul schème globalisant, l'historiographie actuelle sur le XIXe siècle canadien a éclaté en un bouquet luxuriant de pistes d'investigation qu'il est impossible de toutes survoler dans le cadre d'un simple article ${ }^{40^{\mathrm{a}}}$. Aussi convient-il davantage d'illustrer la richesse au moins potentielle des percées récentes dans certaines directions caractéristiques et d'en extraire les grands patterns: ces derniers englobent une multitude de champs nouveaux (extension), presque tous situés au niveau des segments ou des régions, et témoignent d'une spécialisation accrue (approfondissement), où on peut lire "le bon usage" des controverses et aussi discerner certaines zones peu actives.

40 C'est ce que suggère $C$. Berger dans la conclusion de son étude, sans toutefois souligner les riches possibilités implicites du cadre de référence trop flou de Careless (voir J.M.S. Careless, "Limited Identities in Canada" et "Nationalism, Pluralism and Canadian History", Culture, 30, 1969). Dans la même veine, on peut se référer également aux schèmes de relations centre-périphérie et externes-internes proposé par W.T. Easterbrook afin d'expliquer le changement dans les socio-économies régionales ("The Entrepreneurial Function in Relation to Technological and Economic Change", B.F. Hozelitz et W.E. Moore, ed., Industrialization and Society (Paris, 1968); l'évocation que fait A. Faucher d'un ensemble de régions imbriquées autour des Grands Lacs; la théorie du développement du sous-développement, utilisée par S. Amin et G. Frank pour les pays sous-développés dans le monde contemporain, et adaptée avec plus ou moins de bonheur par A. Dubuc ("Les inégalités économiques", Le Devoir, 2 et 3 mars 1973) et N. Séguin (La conquête du sol au 19e siècle (Montréal, 1977); le procès d'affaiblissement du capital industriel soumis aux contraintes d'un capital commercial persistant (T. Naylor, History of Canadian Business, 1867-1914, 2 vol. (Toronto, 1975); avec quelques-unes des précédentes, diverses variantes marxistes et marxiennes, parfois limitées par des postulats théoriques trop rigides ou l'absence de données empiriques suffisamment riches (S. Ryerson, Le capitalisme et la Confédération [...] (Montréal, 1972); G. Bourque, Classes sociales et question nationale au Québec, 1750-1840 (Montréal, 1970); l'hypothèse "frontière" (M. Cross, The Frontier Thesis and the Canada [...] (Toronto, 1970).

40a On trouvera des survols utiles de l'historiographie portant sur le XIXe siècle canadien dans H.J. Hanham, "Canadian History in the 1970's"; J.L. Granatstein et P. Stevens, ed., Canada since 1867 (Toronto, 1977); P.S. Soroko, "A Soviet Critique of the Canadian Historical Review", CHR, 57, 1976; J.M.S. Careless, "The Review Reviewed or Fifty Years with the Beaver Patrol", CHR, 51, 1970. 
Le défrichement de nouveaux champs de recherche n'est pas attribuable au seul accroissement du nombre d'historiens, mais surtout au surgissement de questions et de perspectives nouvelles ou plus aiguisées. L'histoire urbaine constitue peut-être le champ qui a suscité le plus grand nombre d'études récentes: on tente d'y déchiffrer certains des sous-procès (démographie, production et échange, écologie des groupes, etc.) qui tissent l'expérience multiforme du Canada ${ }^{41}$. L'histoire ouvrière connaît également une

41 Pour des études bibliographiques et historiographiques sur la question, voir: G.A. Stelter, "The Historian's Approach to Canada's Urban Past", Histoire sociale [HS], 7, 1974; J.-L. Granatstein et P. Stevens, Canada since 1867, 88-89, 92, 122-123, 146ss, 195; P.A. Linteau et al., Montréal au 19e siècle, blibliographie (Montréal, 1972); G.A. Nader, Cities of Canada, I: Theoretical, Historical and Planning Perspectives (Toronto, 1975). La revue Histoire urbaine [HU], publiée par le Musée National de l'Homme depuis 1972, comporte des notices bibliographiques et des articles monographiques (voir, par exemple, la livraison de juin 1975 qui étudie sept grandes villes canadiennes, de Halifax à Vancouver). Le Musée a aussi inauguré la publication de monographies d'histoire urbaine (v.g. A. Artibise, Winnipeg. An Illustrated History (Toronto, 1977). Voir aussi, dans ce cas comme pour les autres thèmes, les Communications historiques de la Société historique du Canada [CHSHC].

Depuis la fin des années 1960, l'accent s'est porté sur les aspects quantifiables de la vie urbaine, sans qu'émerge un cadre conceptuel clair qu'aurait accepté une majorité d'historiens. La plupart des monographies récentes s'intéressent aux procès et aux événements dans la ville: surtout les procès socio-économiques et le développement, vus parfois "as the interaction of the decision-making of dynamic individuals or groups and technological and population change" (Stelter, op. cit., 11); il cite des études de Careless, Tulchinsky, Artibise, Armstrong, Acheson, Klassen, Macmillan, Ouellet, McCala, Chambers, Bertram et Faucher. Pour cette tendance, voir aussi B. Young, "Railway Politics in Montreal, 1869-1878", CHSHC, 1972; A.R. McCormack et I. MacPherson, Cities in the West [...] (Ottawa, 1975); A.W. Rasporich et H.C. Klassen, ed., Frontier Calgary: Town City and Region 1875-1914 (Calgary, 1975); aussi l'histoire sociale, i.e., la démographie, la stratification sociale, les structures d'âge et de fortunes, la répartition géographique, les conditions de vie, les moeurs, etc. (les études de M. Katz et du GRSM, dont nous reparlerons plus loin; celles de F. Ouellet sur Québec et Montréal dans Éléments d'histoire sociale (Montréal, 1971); de N. MacDonald sur Vancouver, in Pacific Historical Review, 39, 1970; de J.I. Cooper et G.E. Hart sur les sociétés de bienveillance à Québec et à Halifax, CHR, 30, 1949, et CHR, 34, 1953; de P. Rutherford sur le mouvement de réforme urbaine, CHSHC, 1971; de F.T. Denton et P.J. George sur Hamilton, HS, 5, 1970; de A.F. Artibise sur Winnipeg, HS, 9, 1976; de J. Hare sur Québec, HS, 7, 1974; de J.-C. Robert (sa thèse) sur Montréal 1821-1971; de T. Copp sur la Classe ouvrière et pauvreté à Montréal (traduction de son livre de 1974) (Montréal, 1978); de F. Fingard sur la pauvreté en ANB avant la Révolution industrielle, CHSHC, 1973); la politique urbaine (v.g. les travaux de G. Bourassa sur l'élite politique de Montréal, L.D. Feldman et M.D. Goldrick, ed., Politics and Government of Urban Canada (Toronto, 1969); de P. Romney sur W.L. Mackenzie, maire de Toronto, CHR, 51, 1975; de D. Sutherland sur la réforme urbaine à Halifax, CHSHC, 1972; de J.-C. Robert sur l'élite montréalaise, HS, 8 , 1975) et les aspects physiques (voir Stelter, "The Historian's Approach", 15-18). D'autres études traitent de la ville "as a special kind of social environment with unique internal and external patterns of organization" (Stelter, "The Historian's Approach", 18; J.T. Lemon, "Study of the Urban Past: Approaches by Geographers", CHSHC, 1973). L'étude de la croissance urbaine en tant qui telle caractérise des oeuvres plutôt rares, telles celles de P. Goheen, Victorian Toronto, 1850 to 1900: Patterns and Process of Growth, 1970, et A. Artibise, Winnipeg: A social History of Urban Growth, 1874-1914, 1975. 
expansion remarquable, d'autant plus qu'on fouille le XIXe siècle pour mieux y saisir la discontinuité de l'industrialisation et son impact sur la montée de la classe ouvrière ${ }^{42}$. L'histoire économique exigerait de longues illustrations, en particulier en ce qui a trait au développement industriel, considéré souvent dans un contexte régional ou en relation avec des secteurs de développement ou de sous-développement, à l'entrepreneurship et aux affaires, au capital

42 J. L. Granatstein et P. Stevens, ed., Canada since 1867, 80-83, 172-173. Bien qu'une partie des travaux cherchent davantage à mousser la "pratique révolutionnaire" qu'à assurer une analyse sérieuse, l'accumulation des études devrait nourrir dans un proche avenir une synthèse préliminaire sur les travailleurs et les mouvements ouvriers tout au long du XIXe siècle, en insistant sur des thèmes tels le rapport travailleurs-technique, la division croissante du travail et la différenciation des groupes sociaux à mesure que le Canada s'industrialise, la protestation sociale et l'action organisée. En fait, l'histoire des travailleurs est inséparable de l'histoire de l'évolution de la structure des classes au Canada. Voir G.S. Keely et P. Warrian, ed., Essays on Canadian Working Class History, 1860-1930 (Kitchener, 1973); J.D.T. Thwaites, Le monde ouvrier au Québec: bibliographie rétrospective (Montréal, 1973); $\mathrm{R}$. Comeau, "L'histoire ouvrière au Québec: quelques nouvelles avenues", RHAF, 28, 4 (mars 1975); H. Espesset et al., "Le monde du travail au Québec aux 18e et 19e siècles [...]", RHAF, 25, 4 (mars 1972); E. Forsey, The Canadian Labour Movement, 1812-1902 (Ottawa, 1974); R. Babcock, Gompers in Canada [...] (Toronto, 1974); T. Copp, Classe ouvrière et pauvreté à Montréal; G. Kealy, ed., Canada Investigates Industrialism [...]1889 (Toronto, 1973); idem, “The 'Honest Workingman' and Worker's Control [...], 1860-1892", Labour/Le Travailleur, 1976; J. Hamelin, éd., Les travailleurs québécois, 1851-1896 (Montréal, 1973); J. Rouillard, Les syndicats nationaux au Québec de 1900 à 1930 (Québec, 1979), chapitres I et 11; C. Lipton, Histoire du syndicalisme au Canada, 1827-1859 (Montréal, 1976); J. de Bonville, JeanBaptiste Gagnepetit: les travailleurs montréalais à la fin du XIXe siècle (Montréal, 1975); F. Harvey, éd., Aspects historiques du mouvement ouvrier au Québec (Montréal, 1973) et la thèse de $\mathrm{Ph}$. $\mathrm{D}$. de Harvey sur l'enquête de la Commission royale à propos des relations entre le capital et le travail entre 1886 et 1889 (Université Laval, 1977); idem, "Une enquête ouvrière au XIXe siècle [...]", RHAF, 30, 1 (juin 1976); R. Tremblay, La nature des procès de travail à Montréal entre 1790 et 1830, thèse de M.A. (Université de Montréal, 1979); idem, "La formation matérielle de la classe ouvrière à Montréal entre 1790 et 1830", RHAF, 33, 1 (juin 1979); J. Scott, Sweat and Struggle: Working Class Struggles in Canada, 1789-1899 (Vancouver, 1974); M. Robin, "Determinants of Radical Labour and Socialist ' Politics in English Speaking Canada between 1880 and 1930", Journal of Canadian Studies [JCS], 2, 1967; M.S. Cross, ed., The Workingman in the Nineteenth Century (Toronto, 1974); idem, "The Shiner's War: Social Violence in the Ottawa Valley in the 1830's", CHR, 54, 1973; S. Langdon, "The Emergence of the Canadian Working Class Movement, 1845-1875", JCS, 8, 1973; les études de G.S. Kealy sur les Chevaliers de St-Chrispin, CHSHC, 1973; de M. J. Piva sur l'“aristocratie du travail", HS, 7, 1974; de J.-P. Kesteman sur les travailleurs des chemins de fer dans la région de Sherbrooke, 1851-1853, RHAF, 31 , 4 (mars 1978); de M. Heap sur la grève des charretiers à Montréal en 1864, RHAF, 31, 3 (décembre 1977).

Sur les métiers, les outils, les apprentis au Québec au 19e siècle, voir J.-P. Hardy et T. Ruddell, Les apprentis artisans à Québec 1660-1815 (Montréal, 1977); la thèse de H.-P. Audet sur les apprentis à Montréal (1792-1812) (Université Condordia, 1973); les articles de J. Bernier sur les outils et les maisons des menuisiers-charpentiers à Québec, RHAF, 31, 4 (mars 1978) et ses brochures au Musée de l'homme, 1976; les textes de G. Paquet et J.-P. Wallot sur les "Groupes sociaux et pouvoir dans le Bas-Canada [...]", RHAF, 27, 4 (mars 1974) et "Les inventaires après décès à Montréal [...]”, ibid., 30, 2 (septembre 1976); la liste confectionnée par J. MacKinnon des ébénistes de Toronto et de Kingston (Musée de l'homme, 1976). 
financier, etc. ${ }^{43}$ L'histoire des femmes, un champ en émergence, nécessitera encore beaucoup de recherches et de travaux, d'ailleurs difficiles à mener, avant que ne se dessine un pattern plus global qui

43 Il est impossible de mentionner ici tous les sous-champs et tous les articles, monographies, etc. En général, on peut dire que malgré de brillantes exceptions, l'histoire économique demeure traditionnelle, descriptive, marquée par une quantification qui atteint rarement le niveau affiné de la "nouvelle histoire économique" des États-Unis. Voir J.L. Granatstein et P. Stevens, Canada since 1867, chap. III, 11-12, 107, 145ss, 168ss, 191ss. Bien que Hanham laisse entendre que l'histoire industrielle est sous-développée ("Canadian History in the 1970's", 19-20), il existe un certain nombre d'études solides sur la croissance industrielle, sur les entrepreneurs, sur les relations entre le monde des affáires et les gouvernements (v.g. H.V. Nelles, The Politics of Development: Forests, Mines àhd HydroElectric Power in Ontario, 1849-1941 (Toronto, 1974); M. Bliss, A living Profit: Studies in the Social History of Canadian Business, 1883-1911 (Toronto, 1974), et idem, The Life and Business Times of Sir Joseph Flavelle, Bart., 1858-1939 (Toronto, 1978); Y. Saint-Germain, The Genesis of the French-Canadian Language Business Press and Journalists in Quebec, $1871-1914$, thèse de $\mathrm{Ph}$. D. (University of Delaware, 1975); la thèse de A. Dubuc sur les Molson; A. Faucher, Histoire économique et unité canadienne (Montréal, 1970) et idem, Québec en Amérique au XIXe siècle (Montréal, 1973); J. Hamelin et Y. Roby, Histoire économique du Québec, 1851-1896 (Montréal, 1971); J. Spelt, Urban Development of SouthCentral Ontario (Toronto, 1973); J. M. Gilmour, Spatial Evolution of Manufacturing in Southern Ontario 1851-1891 (Toronto, 1972); R. Cuff et G. Porter, Enterprise and National Development: Essays in Canadian Business and Economic History(Toronto, 1973); A.R.M. Lower, Britain's Woodyard: British America and the Timber Trade 1763-1867 (Montréal, 1973) - il s'agit de la thèse de doctorat de Lower publiée tardivement; l'ouvrage classique de V.C. Fowke, The National Policy and the Wheat Economy (Toronto, 1957, 1978). Voir aussi: L.A. Wood, A History of Farmers' Movements in Canada: The Origins and Development of Agrarian Protest, 1872-1914 (Toronto, 1975); différents articles de McCalla sur le commerce des céréales dans les années 1840, CHSHC, 1973; de A.A. den Otter sur Galt et le charbon de l'Alberta, CHSHC, 1973; de J.M.S. Careless sur le développement du groupe d'hommes d'affaires à Winnipeg, Transactions of the Royal Society of Canada, 4, 8, 1970; T.W. Atcheson sur la politique nationale et l'industrialisation des Maritimes, Acadiensis, 1, 1972; de D. Sutherland sur les marchands d'Halifax et la recherche du développement (17831850), CHR, 59, 1978; de F. David sur le charbon et l'acier au Cap Breton, Acadiensis, 7 , 1977; de W.A.B. Douglas, "The Ocean in Nineteenth Century Canadian History", C.G. Reynolds et W.J. McAndrews, ed., Proceeding, 1971 Seminar in Maritime and Regional Studies (Orono, 1972); de P. Basherville sur la navigation à vapeur et l'écoñomie hautcanadienne, Ontario History [OH], 67, 1975; de D. McCalla sur Hamilton, OH, 65, 1973; de G. Tulchinsky sur le Richelieu et une compagnie de navigation à vapeur, RHAF, 26, 4 (mars 1973), de même que sa thèse publiée en 1977 sous le titre de The River Barons. Montreal Businessmen and the Growth of Industry ans Transportation, 1837-1853 (University of Toronto Press); de J. Burgess sur l'industrie de la chaussure à Montréal(1840-1870), RHAF, 31, 2 (septembre 1977); de K.H. Norrie, "The Rate of Settlement on the Canadian Prairies, 1870-1911", JEH, 35, 1975; de K. Kelly sur l'influence des techniques britanniques sur l'agriculture haut-canadienne avant 1850, OH, 63, 1971; le livre de N. Séguin, La conquête du sol [...]. Sur la période d'avant 1850, au Québec, voir les livres de F. Ouellet déjà mentionnés (note 33); M. Séguin, La nation "canadienne" et l'agriculture (1760-1850) (Trois-Rivières, 1970); G. Paquet et J.-P. Wallot, "Le Bas-Canada au début du XIXe siècle: une hypothèse”, RHAF, 25, 1 (juin 1971); idem, "La crise agricole et tensions socio-ethniques [...]", RHAF, 26, 2 (septembre 1972); "International Circumstances of Lower Canada [...]", CHR, 53, 1972. 
tienne compte des disparités régionales déjà visibles ${ }^{44}$. Parmi les groupes qui composent la "mosaïque canadienne", on n'a pas négligé les ethnies et plus précisément les relations entre les Amérindiens et les Européens ${ }^{45}$. L'histoire culturelle s'ouvre à un vaste horizon, depuis la culture au sens plus élitiste, les idéologies, les imprimés, l'instruction, etc. ${ }^{46}$ jusqu'aux travaux de plus en plus concertés sur les "genres de vie", la culture matérielle, les loisirs, le

44 Voir J.L. Granatstein et P. Stevens, Canada since 1867, 84, 92-93, 153, 173-174; M. Stephenson, ed., Women in Canada (Toronto, 1973); C. Cleverdon, The Woman Suffrage Movement in Canada (Toronto, 1974); S. Trofimenkoff et A. Prentice, ed., The Neglected Majority: Essays in Canadian Women's History (Toronto, 1977); M. Lavigne et L. Pinard, Les femmes dans la société québécoise, aspects historiques (Montréal, 1977); D.S. Cross, "The Neglected Majority: The Changing Role of Women in 19th Century Montreal", HS, 6, 1973; Women at Work: Ontario, 1850-1930 (Toronto, 1974); R. Pierson, "Women History: The State of the Art in Atlantic Canada" Acadiensis, 7, 1977; S. Van Kirk, "Woman in Between': Indian Women in Fur Trade Society in Western Canada”, CHSHC, 1977; A. Morel, "La libération de la femme au Canada: deux itinéraires", Thémis, 5, 1970; M. DumontJohnson, "Peut-on faire l'histoire de la femme?", RHAF, 29, 3 (décembre 1975).

45 J. L. Granatstein et P. Stevens, Canada since 1867, 88-89. Ces études portent non -seulement sur "la" minorité, i.e. les Canadiens français: M. Séguin, Sociologie du national (miméo, 1965); R. Cook, The Maple Leaf Forever (Toronto, 1971); R. Choquette, Language and Religion: The History of English-French Conflicts in Ontario (St-Boniface, 1973) et les articles de R. Painchaud et A. Silver sur des questions similaires, CHR, 59, 1978 et 57, 1976), mais aussi sur les Amérindiens et les Métis: CHSHC, 1975; Acadiensis, 7, 1977; A.J. Ray, Indians in the Fur Trade (Toronto, 1974); R. Fisher, Contact and Conflict: Indian-European Relations in British Columbia, 1774-1890 (Vancouver, 1978), les Noirs (CHR, 50, 1969; Dalhousie Review, 48, 1968; OH, 61, 1969), les Chinois (JCS, 6, 1971; B. C. Studies, 22, 1974), les Irlandais (Canadian Catholic Hist. Ass. Study Sessions, 39, 1972; J.J. Mannion, Irish Settlements in Eastern Canada, (Toronto, 1973), les Ecossais (R.A. Maclean, Beyond the Atlantic Roar: A Study of the Nova Scotia Scots (Toronto, 1974), les Polonais, etc. Voir A. Gregorovich, Canadian Ethnic Groups Bibliography (Toronto, 1972).

${ }_{46}$ J. L. Granatstein et P. Stevens, Canada since 1867, passim; A. Beaulieu et J. Hamelin, La presse québécoise des origines à nos jours, tome I; 1764-1859 (Québec, 1973), tome II: 1860-1880 (Québec, 1975), tome III: 1880-1895 (Québec, 1977); Y. Lamonde, "La recherche sur l'histoire de l'imprimé et du livre québécois", RHAF, 28, 3 (décembre 1974); J. Hare et J.-P. Wallot, Les imprimés dans le Bas-Canada, 1801-1810 (Montréal, 1967); les travaux émanant du Groupe de recherche sur l'histoire des imprimés (notamment ceux de C. Galarneau, J.-L. Roy, Y. Lamonde, etc.); J.-C. Falardeau, Etienne Parent, 1802- 1874 [...] (Montréal, 1975); les articles de P.-L. Lapointe sur les nouvelles européennes dans les journaux québécois, RHAF, 28, 4 (mars 1975); de A. Greer sur l'analphabétisme au Québec, 1745-1899, HS, 11, 1978; de J. de Bonville sur la liberté de la presse à la fin du XIXe siècle, RHAF, 31, 4 (mars 1978); de Y, Lamonde sur les archives de l'Institut canadien, R HAF, 28, 1 (juin 1974); J.-L. Roy, Edouard-Raymond Fabre, libraire et patriote canadien (1799-1854) (Montréal, 1974); aussi D. Monière, Le développement des idéologies au Québec des origines 
folklore, etc ${ }^{47}$. Quelques nouvelles approches aux réalités politiques de cet ensemble de colonies, de sous-régions et de groupes d'intérêt divers, ont affleuré de façon plutôt timide ${ }^{48}$. Et il faut bien enregistrer la recrudescence de ce composé d'un peu tous ces filons qui en confirme la pertinence, soit celle de "l'histoire régionale": en fait, la plupart des études récentes sur la géographie, la population,

à nos jours (Montréal, 1976); idem et A. Vachet, Les idéologies au Québec (Montréal, 1976); J.-P. Bernard, Les Rouges. Libéralisme, nationalisme et anticléricalisme au milieu du XIXe siècle (Montréal, 1971); idem, Les idéologies québécoises au 19e siècle (Trois-Rivières, 1973); F. Dumont et al., Idéologies au Canada français, 1850-1900 (Québec, 1971); les articles de Nadia F. Eid sur l'ultramontanisme, RHAF, 29, 1 (juin 1975) et 32, 1978) et son Clergé et pouvoir politique au Québec (Montréal, 1978); les études déjà mentionnées de Berger et Bliss sur l'idéologie impérialiste et l'idéologie des milieux d'affaires; l'étude d'À. İ. Ŝilver sur l'impérialisme au Québec, CHR, 57, 1976. Aussi: B. Denault et B. Lévesque, Eléments pour une sociologie des communautés religieuses au Québec (Montréal, 1975); l'édition massive (plus de 11 volumes parus en 1973) par le R.P. Carrière, o.m.i., de documents sur les missions oblates; J.E. Rea, Bishop Alexander Macdonell and the Politics of Upper Canada (Toronto, 1974); J. Moir, The Church in the British Era (Toronto, 1972); l'article de R. Hardy sur la montée du protestantisme durant les rébellions de 1837-1838, RHAF, 29, 2 (septembre 1975) et sa thèse de $\mathrm{Ph}$. D. (Laval, 1978) Les zouaves pontificaux et la diffusion de l'ultramontanisme au Canada français, 1860-1870; J. Usher, William Duncan of Metakatla: A Victorian Missionary in British Columbia (Ottawa, 1974); T. Flannigan, Louis 'David' Riel, Prophet of the New World (Toronto, 1978); R. Chabot, Le curé de campagne et la contestation locale au Québec [...] (Montréal, 1975); l'article de J.-P. Wallot sur la religion et les moeurs des Canadiens, CHR, 52, 1971; de L. Chevrette sur l'ultramontanisme, RHAF, 25, 2 (septembre 1971). En matière d'éducation, voir L. K. Shook, Catholic Post-Secondary Education in English-Speaking Canada: A History; M.B. Katz et al., Education and Social History in Canada: Themes from Ontario's Past (New York, 1975); C. Galarneau, Les collèges classiques au Canada français (Montréal, 1978); les articles de R.A. Jarrell sur l'enseignement de la science à Québec (1824-1844), HS, 10, 1977; de S.E. Houson sur la politique, les écoles et le changement social dans le Haut-Canada, CHR, 53, 1972; de R.D. Gidney sur l'opinion publique haut-canadienne relativement aux écoles, HS, 5, 1972; de A. Prentice sur la "féminisation" de l'enseignement dans l'A.N.B.. HS, 8, 1975; aussi A. Lavallée, Québec contre Montréal. La querelle universitaire, 1876-1891 (Montréal, 1974).

47 Voir en particulier le Bulletin d'histoire de la culture matérielle, publié par le Musée National de l'Homme depuis 1976 et plusieurs des études sur les conditions de vie des travailleurs dans la note 42 ; les nombreux livres de R.-L. Séguin, dont La civilisation traditionnelle de l"'habitant" aux 17e et 18e siècles. Fonds matériel (Montréal, 1967); N. Lafleur et al., La vie quotidienne des premiers colons en Abitibi-Témiscamingue (Montréal, 1976); idem, La drave en Mauricie [...] (Montréal, 1970); idem, La vie traditionnelle du coureur de bois au XIXe et au XXe siècle (Montréal, 1973); J.-C. Dupont, éd., Habitations rurales au Québec (Montréal, 1978); les études de Parcs Canada sur le chauffage en NouvelleFrance et dans les Canadas et celles du ministère des Affaires culturelles sur divers métiers traditionnels; les actes à paraître du colloque d'histoire de la culture matérielle au Musée de l'Homme, février 1979; l'article de J.J. Bellomo sur les attitudes envers le crime et le châtiment dans le Haut-Canada, OH, 64, 1972; de G. Paquet et J.-P. Wallot, "Les inventaires après décès [...]", RHAF, 30, 2 (septembre 1976). 
l'économie ou les traits sociaux et ethniques de la société, ainsi que sur sa culture et ses idéologies, ajoutent à un corpus de plus en plus imposant sur les études régionales qui s'inspire d'une nouvelle vision du Canada conçu comme une matrice complexe d'expériences historiques variées, marquées par des rythmes et des caractéristiques différentes de colonisation. On ne se contente plus de voir dans

48 Brown, Cook, Nelles et quelques autres ont réussi à lier les niveaux politique, économique et social. Voir aussi F. Ouellet, Le Bas-Canada 「...]; R. Durocher et al., Histoire du Québec contemporain, tome I: De la Confédération à la crise (Montréal, 1979); J. Levitt, Henri Bourassa on Imperialism and Bi-Culturalism (Toronto, 1970); A. Sweeny, George Étienne Cartier: A Biography (Toronto, 1976); G. Paquet et J.-P. Wallot, Patronage et pouvoir dans le Bas-Canada (1794-1812): un essai d'économie historique (Montréal, 1973) et "Groupes sociaux [...]"; J.-P. Wallot, Un Québec qui bougeait. Trame socio-politique au tournant du XIXe siècle (Montréal, 1973); H. Brun, La formation des institutions parlementaires québécoises (1791-1838) (Québec, 1970); J.-Y. Gravel, L'armée au Québec (1868-1900)(Montréal, 1974); A. Désilets, Hector-Louis Langevin. Un père de la Confédération canadienne (1826-1906)(Québec, 1969); M. Caya, “Aperçu sur les élections provinciales du Québec de 1867 à 1886”, RHAF, 29, 2 (septembre 1975); de nombreux articles dans diverses revues régionales, dont B.C. Studies, Saskatchewan History, Acadiensis et $O H$; celui de W.M. Baker sur la répression anti-fénienne au Nouveau-Brunswick, $C H R, 55,1974$. Peu d'études ont vraiment renouvelé le "genre" en présentant de nouvelles perspectives et en empruntant des outils conceptuels nouveaux à la science politique. D. Morton semble l'avoir réussi pour l'histoire militaire: Ministers and Generals: Politics and the Canadian Militia, 1868-1904 (Toronto, 1970); The Canadian General: Sir William Otter (Toronto, 1974). I1 n'est pas certain que le domaine des relations internationales ait suscité des travaux vraiment nouveaux, même si les monographies solides ont continué à s'accumuler. Voir J. Morchain, Sharing a Continent. An Introduction to Canadian-American Relations (Toronto, 1973); R. A. Preston, ed., The Influence of the United States on Canadian Development: Eleven Cases Study (Durham, 1972). P. Tousignant (La genèse et l'avènement de la constitution de 179I, thèse de $\mathrm{Ph}$. D. (Université de Montréal, 1971) et son article dans la RHAF, 27, 2 (septembre 1973) relie la constitution de 1791 au contexte impérial, notamment à la nature artistocratique de la société anglaise. Voir ausi P. Burroughs, The Canadian Crisis and British Colonial Policy, 1828-1841 (Toronto, 1972). M. Hamelin a rejeté les thèses traditionnelles sur les politiciens québécois et leurs politiques (Les premières années du parlementarisme québécois (1867-1878) (Québec, 1974). Voir aussi E. Forsey, Freedom and Order: Collected Essays (Toronto, 1974); R. Boily, "Les hommes politiques du Québec, 1867-1967", RHAF, 21, 3a (1968); les nombreuses biographies dans le Dictionnaire biographique du Canada; la thèse de M. Caya sur le parti libéral au Québec; R. Rudin, "Regional Complexity and Political Behaviour in a Quebec County, 1867-1886", HS, 6, 1976; M. R. Lupul, The Roman Catholic Church and the North-West School Question [...] (Toronto, 1974); J. R. Noël, Political History of Newfoundland (Toronto, 1971); le livre de Bolger sur l'Ile-du-Prince-Edouard (Charlottetown, 1973); M. Robin, The Rush for Spoils: The Company Province, 1871-1933 (Toronto, 1972); D. E. Smith, Prairie Liberalism [...] (Toronto, 1975); L. H. Thomas, The Struggle for Responsible Government in the North-West Territories 1870-1897, 2e éd., (Toronto, 1978); P. Crunican, Priests and Politicians. Manitoba Schools and the Elections of 1896 (Toronto, 1974). 
le développement de l'Ouest ou du Nord ou des provinces atlantiques de simples effets d'écho passif de décisions et d'évolutions survenues dans les provinces centrales. On dissèque même des sousrégions ${ }^{49}$.

Cet éparpillement apparent s'accompagne d'une spécialisation accrue des recherches, d'un approfondissement du savoir dans ces territoires reliés entre eux par des perspectives parfois convergentes. D'aucuns soutiennent que cette dispersion, jumelée à une quantification plus poussée (sous l'influence des historiographies américaine, anglaise et française, y compris celle des Annales), a poussé les historiens à se barricader dans des ghettos: ghettos de spécialités, de langages spécifiques, d'interprétations préfabriquées et exclusives. En conséquence, l'ensemble de ces travaux récents composerait une tapisserie trop disparate pour qu'on puisse commencer à y distinguer une compréhension plus globale du XIXe siècle canadien. Â notre avis, il s'agit là d'un point de vue simpliste. À la fragmentation de l'effort historique a correspondu une consolidation des arcs-boutants. En témoigne, par exemple, la formation de groupes de recherche organisés. Ces points de ralliement polarisent les efforts de spécialistes aux disciplines complémentaires autour de

49 J. L. Granatstein et P. Stevens donnent une bonne idée de l'historiographie pour les différentes régions du Canada (Canada since 1867). Voir aussi R. Cook, "The Burden of Regionalism", Acadiensis, 7, 1977; F. H. Armstrong et al., ed., Aspects of Nineteenth Century Ontario: Essays Presented to James J. Talman (Toronto, 1974); F. Landon, Western Ontario and the American Frontier (Toronto, 1967); les travaux et rapports du "Landon Group" de recherche, par ex. R.S. Alcorn et J. Igartua, "Du rang à la ville: le processus d'urbanisation au Québec et en Ontario", RHAF, 29, 3 (décembre 1975). Pour l'Ouest, le Nord et les Maritimes, voir M. Zaslow, The Opening of the Canadian North, 1870-1914 (Toronto, 1971); le livre classique de W. L. Morton, Manitoba, 2e éd. (Toronto, 1967); D. C. McGowan, Grassland Settlers: The Swift Current Region during the Era of Ranching Frontier (Regina, 1975); l'étude de P. E. Neary sur Bell Island et Conception Bay, CHSHC, 1973; J. A. Macgregor, A History of Alberta (Edmonton, 1972); G. F.G. Stanley, Manitoba 1870: A Metis Achievement (Winnipeg, 1972); C. Berger et R. Cook, ed., The West and the Nation: Essays in Honour of W. L. Morton (Toronto, 1976); A. W. Raporich, ed., Western Canada: Past and Present (Calgary, 1975); L. H. Thomas, ed., Essays on Western History (Edmonton, 1976); les articles de K. H. Harris sur le rythme de colonisation dans les Prairies, in Journal of Economic History, 35, 1975; de T. W. Acheson sur la politique nationale et les Maritimes, Acadiensis, 1, 1972; de J. A. Lower sur l'Ouest et l'Ontario, in Quarterly of Canadian Studies, 4, 1976; E. Kerr, The Canada Land Company [...] (Toronto, 1974). En Ontario, plusieurs gro!upes scrutent divers comtés (v.g. les projets Landon et Peel County). Il en est de même au Québec où les patterns régionaux varient beaucoup depuis la plaine de Montréal jusqu'à la région de Québec, au Saguenay ou à la péninsule de Gaspé(v.g. le groupe de G. Bouchard à Chicoutimi, celui de R. Hardy et $\mathrm{S}$. Gagnon à Trois-Rivières). 
l'identification de données pertinentes, tâche souvent gigantesque en soi, de leur analyse et de l'élaboration d'études complexes. Ils s'agrègent en un réseau de points nodaux, dont chacun est polymorphe et interdisciplinaire, d'où leur tendance plus accentuée à tenter des généralisations hardies (parfois trop hardies, parfois trop timides) à l'intérieur de leur "région" 50 du savoir.

On ne saurait se pencher sur chacun de ces points nodaux, même de façon superficielle. Granatstein et Stevens ont déjà procédé à une reconnaissance des principales enquêtes historiographiques par région, par spécialité et par approche. Ce qu'il faudrait maintenant, ce sont des comptes rendus spécialisés de l'état de la question pour chacun de ces points nodaux, un peu comme celui de Stelter en ce qui a trait à l'histoire urbaine dans la revue Histoire sociale. Dans le présent article, il suffira de citer quelques expériences intéressantes en cours.

Dans les Maritimes, les recherches du Memorial Group on Maritime History promettent des résultats qu'on n'aurait pas pu envisager il y a à peine une dizaine d'années. En effet, les dépôts d'archives sont si riches sur des sujets tels les pêcheries, la navigation, les navires, les ports, les marins, les entrepreneurs dans la construction navale et les armateurs, qu'ils peuvent alimenter des monographies captivantes pour plusieurs décennies à venir. Les premiers efforts du groupe visent à comparer le développement de plusieurs ports des Maritimes en tenant compte du système atlantique en général et de l'impact des changements techniques.

$\mathrm{Du}$ côté des études urbaines, Michael Katz, membre du Canadian Social History Project, a publié une monographie ponctuelle sur Hamilton. L'auteur spectrographie Hamilton en 1851 grâce à des techniques statistiques relativement affinées; mais ses outils conceptuels n'atteignent pas la même finesse. Quoi qu'il en soit, il scrute la composition de la population, les métiers et professions, les niveaux de richesse et la stratification sociale, la vie familiale, la religion, l'instruction, la physionomie de la ville, son économie, etc. à partir des données des recensements, des registres d'État civil et des archives municipales. Ses conclusions les plus importantes portent sur la mobilité géographique et l'inégalité

50 Pour la notion de "rationalismes régionaux", voir G. Bachelard, Le rationalisme appliqué (Paris, 1949), chap. VII. 
sociales1. À Montréal, le GRSM (“Groupe de recherche sur la société montréalaise”, UQAM) cherche à embrasser tant le développement urbain comme tel de Montréal (évolution physique, fonctions urbaines, sources de croissance, niveaux d'activité économique) que la société qui y vit. Toutefois, cette équipe a opté pour l'échantillonnage en plusieurs cas plutôt que pour le dépouillement exhaustif et l'utilisation de toutes les sources, démarche forcément plus lente adoptée par Katz. Ses travaux préliminaires aident déjà à mieux comprendre la transition de Montréal depuis le stade de ville commerciale, entre 1800 et 1838 , à celui de ville industrielle, entre 1851 et $1871^{52}$.

D'autres groupes observent des régions qui ne sont ni entièrement rurales, ni entièrement urbaines: par exemple, le "Groupe de recherche sur le Saguenay" (à l'Université du Québec à Chicouti$\mathrm{mi})^{53}$, le "Groupe d'histoire régionale de Trois-Rivières" (Université du Québec à Trois-Rivières), le Peel County History Project ${ }^{54}$ et le Landon Project ${ }^{55}$. Le History of Ontario Project ne s'avère pas une recherche intégrée en soi, mais un programme de monographies subventionnées par le gouvernement ontarien ${ }^{56}$. L'Atlas historique

51 Voir les nombreux rapports minéo et les articles de Katz, ainsi que son livre The People of Hamilton, Canada West: Family and Class in a Mid-Nineteenth-Century City (Cambridge, 1976). À l'origine, Katz envisageait d'étudier Hamiltón entre 1851 et 1871. Le coût extraordinaire d'une telle entreprise en argent, en temps et en efforts peut en limiter l'utilité pour des fins de comparaison, d'autant plus qu'il est loin d'être évident que la ville de Hamilton est typique des villes pré-industrielles, qu'elle s'avère une "ville frontière" (G.A. Stelter, "The Historian's Approach [...]", p. 13). En tout état de cause, on ne peut nier les retombées très riches de pareille recherche sur d'autres projets similaires.

52 Le GRSM en est encore à la cueillette et à l'organisation préliminaire des données. Il a estompé quelque peu son cadre théorique un peu rigide du début et a opté pour une stratégie de recherche plus empirique qui ne laisse pas tomber les objectifs initiaux essentiels, dont l'étude des classes sociales. Voir les Rapports du GRSM; J.-C. Robert, "Les notables de Montréal au XIXe siècle"; P.-A. Linteau et J.-C. Robert, "Propriété foncière et société à Montréal: une hypothèse", RHAF, 28, 1 (juin 1974); J.-P. Bernard et al., "La structure professionnelle de Montréal en 1825", RHAF, 30, 3 (décembre 1976); P.-A. Linteau, "Quelques réflexions autour de la bourgeoisie québécoise, 1850-1914", RHAF, 30, 1 (juin 1976).

53 Comme pour le GRSM, les articles sont nombreux. Mentionnons G. Bouchard, “Introduction à l'étude de la société saguenayenne aux XIXe et XXe siècles", RHAF, 31, 1 (juin 1977): 3-27. Il ne faudrait pas négliger l'apport de N. Séguin à l'étude du Saguenay. Outre son livre, voir le numéro spécial de Protée, 4 (printemps 1975) édité par G. Dufour et N. Séguin sur la recherche en études régionales au Saguenay. Séguin s'est joint au groupe de $S$. Gagnon et R. Hardy à l'UQTR qui se penche sur la Mauricie.

54 D. Gagan et H. Mays, "Historical Demography and Canadian Social History: Families and Land in Peel County, Ontario", CHR, 54, 1973.

s5 R.S. Alcorn et J. Igartua, "Du rang à la ville"; Rapports du Landon Project. Series".

s6 Voir les rapports annuels de "Board of Trustees of the Ontario Historical Studies 
$d u$ Canada, malgré son ancrage à l'Université de Toronto, stimulera le surgissement d'équipes de recherche plus petites dans les différentes régions du Canada et permettra sans doute d'élucider certains des sous-procès les plus importants dans l'évolution complexe et différenciée du Canada - l'un des buts explicites de l'entreprise. Le projet démarre et on n'en verra les produits que dans quelques années. Par contre, le CHEQ (Centre d'histoire économique du Québec) a contribué à la publication de plusieurs ouvrages, dont l'étude de Hamelin et Roby sur l'économie québécoise après 1850.

Le "Groupe de recherche sur l'histoire des affaires", présidé par Alfred Dubuc, attire des chercheurs de différentes universités du Québec, tant francophones qu'anglophones ${ }^{57}$. D'autre part, les groupes de recherche sur l'histoire des travailleurs semblent proliférer et se relayer en plusieurs vagues: d'où un certain danger de dispersion des forces ou, peut-être, le risque d'une anarchie créatrice ${ }^{58}$. D'autres enfin font converger leurs efforts autour de l'histoire de la culture matérielle ${ }^{59}$ ou encore de l'histoire du livre, de l'imprimé, des idées ${ }^{60}$.

Bref, ce bouillonnement dans la recherche, dans l'accumulation de matériaux sociographiques et dans la conception d'hypothèses ou de perspectives "régionales" caractéristiques, se reflète

57 Deux membres du groupe viennent de publier un outil de travail fort précieux: $\mathbf{R}$. Rice et B. Young, Guide pour l'étude d'entreprises montréalaises et de leurs archives avant 1947 (Montréal, 1979).

$58 \quad \mathrm{Au}$ Québec seulement, plusieurs groupes se concentrent sur l'histoire ouvrière aux universités du Québec à Montréal et à Rimouski, à l'Université Laval, à l'Université de Montréal. Certains sont "locaux" ou provinciaux, d'autres ont des liens pan-canadiens. Le "Groupe de recherche sur l'histoire des mouvements politiques des travailleurs" (UQAM) a publié une Chronologie des mouvements politiques ouvriers du Québec de la fin du 19e siècle jusqu'à 1919 (Montréal, 1976). Le comité sur l'histoire des travailleurs canadiens publie une revue annuelle, Labour / Le Travailleur depuis 1976.

59 Différents groupes existent au Musée National de l'Homme à Ottawa, à l'UQTR (sous la direction de R.-L. Séguin), à l'Université Laval (notamment au CELAT sous la direction de L. Lacourcière, J. Hamelin et J.-C. Dupont), en plus d'un groupe spécialisé en histoire orale à l'UQAM.

60 C. Galarneau, J.-L. Roy, Y. Lamonde et plusieurs autres forment un groupe informel d'étude des livres et des imprimés. Le GRISCAF ("groupe de recherche sur les idéologies au Canada français") a publié les communications présentées à son colloque sur le "Canada et la Révolution atlantique", Annales historiques de la Révolution française, 45, 1973. P. Savard dirige le Centre de recherche sur la civilisation canadienne-française à l'Université d'Ottawa et est co-éditeur des oeuvres complètes de F.-X. Garneau. Il y a aussi le "Linguistic Survey of the Ottawa Valley"(E. Padolsky et I. Pringle) à l'Université Carleton. 
depuis déjà plusieurs années dans la production historique ellemême: glissement au niveau du contenu des revues spécialisées, naissance de nouvelles revues ${ }^{61}$. La masse d'historiens toujours plus nombreux, leurs préoccupations et leurs liens parfois souterrains avec divers points nodaux, l'accumulation même des nouvelles études, ont amorcé une seconde ronde d"impatience". D'où l'adoption et l'accréditation parfois trop rapide de certaines problématiques trop simples ou la multiplication d'emprunts aux chercheurs d'Europe (notamment ceux des Annales) et d'Amérique latine. Il ne faut donc pas s'étonner de ce que des programmes de recherche concurrents, enracinés dans des problématiques partielles différentes et même parfois antagoniques, aient déclenché des controverses fort vives. Controverses dont l'historiographie semble pourtant faire un bon usage: par exemple, celle autour de l'existence d'une crise agricole au début du XIXe siècle, de la nature de cette crise présumée et de ses effets, alors que les problématiques et les conclusions diffèrent notablement 62 ; l'affrontement entre ceux qui affirment l'émergence du capitalisme industriel dans le Canada central au cours de la seconde moitié du XIXe siècle, et ceux pour qui la persistance du capital marchand a étouffé son développement ${ }^{63}$; les débats entre ceux qui attribuent à la montée de

61 Voir la note 40a. Ces glissements apparaissent clairement. On n'a qu'à comparer le livre de R.C. Brown et de R. Cook, Canada, 1896-1921: A Nation Transformed (Toronto, 1974), avec les études qui l'ont précédé dans la série du Centenaire: Brown et Cook s'intéressent aux procès socio-économique et politique tout autant qu'aux changements structurels, alors que leurs prédécesseurs dans la série suivent l'approche traditionnelle, i.e. politique et narrative. Dans la RHAF, une comparaison entre les années 1955-1963 et les années 1963-1972 révèle une montée appréciable de l'espace dévolu aux articles socioéconomiques et aux sujets d'histoire intellectuelle, une croissance extraordinaire des espaces consacrés à la période post-1867 et à l'histoire du Québec français (F. Harvey et P.-A. Linteau, "L'évolution de l'historiographie dans la Revue d'histoire de l'Amérique française 1947-1972", RHAF, 26, 2 (septembre 1972): 163-183). La naissance de revues comme HS, HU, Le Travailleur et plusieurs autres périodiques spécialisés au cours des dernières années reflète ces changements.

62 Paquet et Wallot opposent la problématique de Mauro et de North, qui voient la source du dynamisme des économies modernes dans le commerce, à l'approche de Ouellet et Le Goff qui est labroussienne dans son accent sur la production agricole (Paquet et Wallot, "Crise agricole"; voir aussi la position de Le Goff et la réplique de Paquet/Wallot dans la CHR, 55, 1974 et 56, 1975).

${ }_{63}$ La thèse de $\mathrm{T}$. Naylor au sujet de l'inexistence ou de la lente émergence du capitalisme industriel (exprimée plus récemment dans son History of Canadian Business, 1867-1914), a été fortement mise en doute par L. Macdonald ("Merchants against Industry: An Idea and its Origin", CHR, 56 (1975): 282-302) et S.W. Langdon (The Political Economy of Capitalist Transformation; Central Canada from the 1840's to the 1870's, thèse de M.A. (Université Carleton, 1972). 
l'économie du blé, vu comme produit générateur à la Innis, un rôle fondamental dans la croissance économique et la hausse du bienêtre au tournant du XXe siècle, et ceux qui ne lui reconnaissent qu'un apport mineur ${ }^{64}$. Dans le bouillonnement actuel, on ne peut prédire si ces pas en avant dans la connaissance seront cumulatifs ou si, comme une multitude de ruisseaux parallèles ou divergents de rétrodictions-explications, leurs cours se perdront sans possibilité de convergence.

Malgré les progrès impressionnants de l'historiographie sur le XIXe siècle canadien, suite à la fragmentation et à la spécialisation de la recherche ainsi qu'à ses consolidations autour de points nodaux interdisciplinaires, on peut déceler de nombreuses zones inactives qu'il faudra animer avant de s'approcher de cet idéal d'une histoire forte proposée par Lévis-Strauss.

1) Au-delà des synthèses fort générales de Innis, Faucher, Warkentin, Harris et quelques autres, l'historiographie manque de travaux fondamentaux sur l'oekoumène. Lacune étonnante au moment même où se répand l'histoire régionale ${ }^{65}$. De même, à quelques exceptions près, les études démographiques sur le XIXe siècle n'ont guère progressé66.

64 Voir E. J. Chambers et D. F. Gordon, "Primary Products and Economic Growth An Empirical Measurement", Journal of Political Economy [JPE], 74 (1966): 315-322; J.H. Dales et al., "Primary Products and Economic Growth: A Comment", JPE, 75 (1967): 881885; G. W. Bertram, "The Relevance of the Wheat Boom in Canadian Economic Growth", Canadian Journal of Economics, 6 (1973): 545-566.

65 Dans Canada before Confederation (New York, 1974), une exception récente, R.C. Harris et J. Warkentin lient l'environnement physique aux caractéristiques et aux développements démographiques, ethniques, techniques et économiques, avec un fort biais du côté de l'expérience historique particulière à chaque région canadienne. L'Atlas historique $d u$ Canada devrait renouveler l'intérêt pour ce champ.

66 Bien qu'on utilise massivement les recencements, surtout à compter de 1841, il n'y a aucune étude démographique sur le XIXe siècle qui puisse se comparerà celle que poursuivent H. Charbonneau et J. Légare sur les XVIIe et XVIIIe siècles. Cependant, certains auteurs, comme Katz, Gagan et Bouchard, utilisent des données démographiques relativement complètes qui seraient encore plus utiles si d'autres études démographiques de même importance étaient conduites pour d'autres régions. Sur les migrations et les taux démographiques usuels au Québec, on trouve peu d'apports nouveaux hormis pour des révisions surtout mineures (voir l'étude de Y. Lavoie sur l'émigration des Canadiens français en Nouvelle-Angleterre in H. Charbonneau, éd., La population du Québec: études rétrospectives (Montréal, 1973); les estimés de J. Henripin et J. Peron sur les taux démographiques du milieu du XVIIIe à la fin du XIXe siècles, ibid.) par rapport à des études comme celles de A. Faucher et G. Paquet dans Recherches sociographiques, 5, 1964. Quelques articles méthodologiques par G. Bouchard et A. La Rose ont aussi été publiés dans la RHAF, 30, 1 (juin 1976) et HS, 8 (1975). 
2) Très peu d'études approfondissent le monde 2 (valeurs, plans, etc.) sauf pour quelques excursions inattendues à l'occasion de biographies, pour des postulats plus ou moins explicites sur les mentalités ou encore pour de vagues synthèses générales ${ }^{67}$.

3) Les travaux sur les structures institutionnelles, sur les arrangements et la façon dont la réalité socio-matérielle canadienne s'est "instituée" au sens de Polanyi, manquent toujours de racines bien plantées dans les "faits matériels" et les "faits moraux". Les historiens n'ont guère mis à jour, selon l'expression de Frédéric Mauro, les structures du système et les mécanismes qui sous-tendent ces structures ${ }^{68}$.

4) Enfin, l'état de la méthodologie dans l'historiographie canadienne n'autorise guère un optimisme débordant. Le débat entre historiens et spécialistes des autres sciences humaines n'a pas été résolu. Nous voilà donc reconduits, en quelque sorte, à la tradition interdisciplinaire de l'économie politique à laquelle renvoie d'ailleurs Carl Berger à la fin de son livre ${ }^{69}$. Se pourrait-il que l'on puisse extraire de cette vieille tradition canadienne des éléments pour une pratique historique mieux ajustée au matériau et dont le besoin se fait tant sentir?

En somme, ce dont les historiens canadiens ont besoin, c'est d'une problématique spécifique permettant d'aborder le Canada à la fois dans ses liens avec l'extérieur - il est un fragment ouvert du système atlantique, une colonie profondément influencée dans sa structuration par les intrusions de l'Empire britannique et l'impact du développement américain - et dans sa dymanique interne, qui est liée aussi aux caractéristiques de la combinaison particulière des

67 Une des rares exceptions: F. Ouellet, Julie Papineau. D'autre part, trop d'historiens utilisent les mentalités comme un dernier recours dans l'explication quand on ne débusque pas d'autre cause. Ou encore, les mentalités deviennent une référence incidente. Une autre exception: R. Chabot, Le curé de campagne [...].

68 Un simple exemple: "The central question is: Why and how have cities grown? This cannot be answered without asking how particular cities connect with one another in a hierarchy or some arrangement of sizes and functions, and how they are distributed over regions as nodes of activity. Also important here historically are the demographic problems of rural/ urban differentia!s and of mobility; rural to urban; urban to urban; and international movements." (J.T. Lemon, "Study of the Urban Past: Approaches by Geographers"). On pourrait poser des questions similaires pour toutes les "régions" de l'exploration historique.

${ }_{69}$ C. Berger, The Writing of Canadian History, 261. 
secteurs qui composent la trame de sa socio-économie (patterns complexes centre-périphérie, etc.). Une telle problématique devrait permettre à la fois de discerner les discontinuités majeures dans le procès socio-économique global, et de rendre compte du fait que ni toutes les régions du pays ni tous les groupes qui en forment le tissu social n'ont éprouvé ces transitions au même moment, ou avec la même intensité, ou comme résultat du même noeud complexe de forces internes et externes. L'accent porterait alors sur les écarts, l'hétérogène, un peu comme dans la voie tracée par la troisième génération des Annales.

\section{VERS UNE PROBLÉMATIQUE MÉSO-ANALYTIQUE}

Ce besoin, ressenti de plus en plus vivement, explique pourquoi l'historiographie récente témoigne d'une désaffection tant pour les grandes perspectives macroscopiques que pour les études de cas au microscope. Au cours de la dernière décennie, l'intérêt s'est déplacé vers des réalités intermédiaires, ce "middle ground" dont parlait Peter McClelland ${ }^{70}$ - groupes, régions, sections, etc. de la socio-économie canadienne du XIXe siècle.

Quel que soit l'intérêt de ces histoires du "middle ground", elles demeurent tributaires d'un découpage naïf des faits humains, d'un découpage qui colle aux faits vécus et à l'expérience immédiate, mais qui s'arrête trop souvent à la surface des choses ${ }^{71}$. Secteur, région, groupe, ville y sont trop considérés comme des phénomènes sui generis. Par conséquent, ils tournent court, ces efforts pour faire que ces études parcellaires s'imbriquent en un portrait du procès socio-économique global.

L'approfondissement de l'analyse exige un découpage moins mécanique, qui suggère des unités d'analyse et un explicandum à un niveau qui permette d'ausculter les sources et les causes de l'évolution du tissu régional/ethnique/sectionnel/etc. de la socioéconomie canadienne. À notre avis, la méso-analyse rend possible un tel découpage des phénomènes.

70 P.D. McClelland, Causal Explanation in History, Economics, and the New Economic History (Londres, 1975), 243. En conclusion à son livre, Berger a noté ce besoin de travailler à un niveau moins agrégatif.

71 Pour une discussion du problème du découpage des phénomènes dans les sciences humaines et des dangers de découpages naïfs, voir G.G. Granger, op. cit., chap. IV. 


\section{a. La méso-analyse}

Dans nos travaux sur le tournant du XIXe siècle, nous avons adopté une définition de la socio-économie canadienne comme "procès institué" 2 . Nous nous sommes attachés à surprendre et à décrire la dynamique de ce procès évolutif en privilégiant la dimension institutionnelle/informationnelle des phénomènes ${ }^{73}$. La question centrale était et demeure celle de savoir quelles sont les forces qui ont fait que ce procès s'est institué différemment d'une région ou section à une autre, d'une période à une autre.

Nous avons emprunté à Michel Crozier l'idée de représenter un procès comme un jeu avec ses acteurs, ses règles, ses frontières, son rythme ${ }^{74}$. On dira que la nature du jeu change quand une socioéconomie s'institue différemment et que survient une rupture ou une discontinuité.

On peut décomposer ce grand jeu en un certain nombre de sous-jeux séparables, chacun avec plus ou moins ses règles propres, sa nature propre. Ils composent, dans leur imbrication, le grand jeu. Il devient possible d'analyser l'évolution de ce grand jeu à partir de l'évolution des sous-jeux, lesquels sont évidemment soumis aux aléas des chocs de l'extérieur et aussi aux effets d'interaction entre eux-mêmes ${ }^{75}$.

La nature des sous-jeux n'est pas figée: à tout moment, la nature du jeu est définie par les acteurs, leurs visions du monde, leurs valences, les règles qui assignent à chacun la structure de ses droits et déterminent la séquence des opérations, et par le système informationnel qui crée le réseau de communication entre acteurs.

72 K. Polanyi, "The Economy as Instituted Process", in K. Polanyi, C.M. Arensberg et H.W. Pearson, ed., Trade and Markets in the Early Empires (New York, 1957), 243-270.

73 Il s'agit d'une constante que nous avons reprise dans nos travaux depuis 1969: G. Paquet et J.-P. Wallot, "Canada 1760-1850: anamorphoses et prospective", in R. Comeau, éd., Economie québécoise (Montréal, 1969); aussi G. Paquet et J.-P. Wallot, "The Agricultural Crisis in Lower Canada [...]", 156ss. Pour une discussion des rapports information/institution, voir G. Newman, "An Institutional Perspective on Information", International Social Science Journal, 28 (1976): 466-492.

${ }_{74}$ M. Crozier, "The Relationship between Micro and Macrosociology", Human Relations, 25, 3 (juillet 1972): 239-251; M. Crozier et E. Friedberg, L'acteur et le système (Paris, 1977); voir aussi N. Georgescu-Roegen, The Entropy Law and the Economic Process (Cambridge, 1971), 211 ss.

75 Il s'agit bien d'une application au monde des organisations d'un procédé bien connu de décomposition utilisé par les spécialistes de recherche opérationnelle. Voir F. Bessière, "The Concept of Separability and the Optimization of Economic Organization", European Economic Review (automne 1969), 74-91. 
A mesure que le jeu se déroule, les règles et les institutions contraignent l'activité des acteurs et les communications entre eux; mais à mesure qu'ils développent des stratégies nouvelles, les flux d'informations muent et il y a lente érosion ou transformation des règles du jeu, voire de la nature du jeu lui-même. Crozier parle en raccourci d'un passage du "jeu féodal" au "jeu capitaliste" comme un exemple de changement dans la nature du jeu socio-économique $^{76}$.

L'unité d'analyse devient dans ce contexte le sous-procès ou le sous-jeu avec ses acteurs, ses règles, ses frontières, son rythme et sa trame organisationnelle. Reste à savoir quels sont les sous-jeux fondamentaux séparables, comment on peut les décrire et les définir, et comment ils peuvent être composés en une image de la socio-économie globale ${ }^{77}$.

\section{b. Six sous-procès fondamentaux}

Notre découpage du procès global en sous-procès ou sous-jeux fondamentaux s'inspire des analyses de John Akerman qui, dès les années 1940, faisait figure de pionnier en ce domaine en tentant de synthétiser le développement des économies occidentales dans une perspective séculaire à l'aide d'une série de sous-procès qu'il nommait "forces motrices" 78 . Nous n'avons repris son découpage

76 M. Crozier et E. Friedberg, op. cit., 332. Pour un examen de la dynamique d'adaptation réciproque des institutions et des réseaux de communication et d'information, voir: G. Newman, op. cit.; G. Paquet, "The Regulatory Process and Economic Performance", G.B. Doern, ed., The Regulatory Process in Canada (Toronto, 1978), 37-38. Les études sur le développement économique présentent beaucoup d'exemples de transformations des règles du jeu ou de l'acquisition de nouveaux traits ou de nouvelles caractéristiques par un jeu ou un procès (v.g. I. Adelman, "Social and Economic Development at the Micro Level - A Tentative Hupo-thesis", E.B. Ayal, ed., Micro Aspects of Development (New York, 1973); A.O. Hirschman, Development Projects Observed (Washington, 1967), chap. IV).

${ }_{77}$ L'étiquette "méso-analyse" suggère donc bien davantage qu'une position intermédiaire entre la petite et la grande échelle au niveau des agrégats. L'unité d'analyse elle-même est modifiée et l'accent porte sur les "systems of inter-relationships, institutional factors and multidimensionality within which margins and marginal analysis do have their proper place in the context of systems" (A. Nove, Efficiency Criteria for Nationalized Industries (Toronto, 1974), 134-135). L'unité d'analyse devient le procès et l'analyse à ce niveau cherche à dégager des schèmes interprétatifs des structures, les règles du jeu, "the rules of operation and structure of institutions for the system as a whole rather than the decision rules of individuals within the system" (I. Adelman, "Social and Economic Development at the Micro-Level $[\ldots] ", 4)$.

8 J. Akerman, Ekonomisk Teori, II (Lund, 1944). Une traduction française a été publiée à Paris en 1955 sous le titre de Structures et cycles économiques. Voir en particulier le vol. I, chap. I et II. 
tel quel. Le nôtre constitue plutôt une reformulation et une condensation des huit forces motrices qu'il identifiait, en six sousprocès ou sous-jeux fondamentaux:

(1) le sous-procès démographique

(2) le sous-procès de production et d'échange

(3) le sous-procès financier

(4) l'écologie des groupes sociaux et leurs motivations

(5) le sous-procès étatique

(6) le sous-procès de répartition.

Nous ne prétendons pas que ces six sous-procès épuisent la richesse de la socio-économie comme procès institué ni qu'ils sont les seules composantes à l'origine du changement dans le grand jeu. Mais, comme le disait Akerman à propos de ses forces motrices, "on peut affirmer avec sécurité qu'elles sont les plus importantes et qu'une reconstitution théorique doit les prendre en considération avant toute autre"79. Ces sous-procès soulèvent en fait l'ensemble des questions au coeur de notre problématique.

Ces six sous-procès ont aussi le mérite d'être assez facilement repérables et de fonctionner avec une régularité suffisante pour qu'on puisse clairement les décrire et les calibrer. Chacun comporte ses acteurs bien définis, sa trame organisationnelle et sa dynamique propre. On peut d'ailleurs espérer en arriver un jour à représenter les règles d'opération de ces sous-procès identifiés plus formellement, par un équivalent de ce que les statisticiens appellent une "fonction génératrice caractéristique" ("characteristic generating function") $)^{80}$.

79 Ibid., 28.

$80 \quad$ L'expression est utilisée par les statiticiens pour désigner la fonction qui engendre tous les termes d'une série. Emery et Trist ont tenté d'étendre la notion à une série d'états d'un procès ou d'un système en acte: "The underlying notion here is that in so far as a system generates its successive phases, it will exhibit some temporal series of behavior which, if quantified, could be represented by a mathematical series. Such a mathematical series has the property that its characteristic generating function can be identified from a finite part of the series (even if the series is infinite), and given the characteristic generating function, one can predict from any starting point the subsequent members of the series". (F.E. Emery et E.L. Trist, Towards a Social Ecology (New York, 1973)passim). Une bonne partie du livre cherche à identifier ou à esquisser des fonctions génératrices caractéristiques pour certains sousprocès socio-techniques importants en vue de prédire leur dérive dans l'avenir. Le langage nécessaire à l'analyse de procès a d'ailleurs été déjà developpé par les cybernéticiens(W.R. Ashby, Introduction to Cybernetics (Londres, 1956). 
Même s'il n'est pas encore possible de formaliser aussi rigoureusement les procès sociaux, on peut en représenter le fonctionnement et en découvrir les règles d'opération ${ }^{81}$. D'ailleurs, dans l'historiographie existante, on peut déjà rassembler des matériaux importants pouvant servir de point de départ à l'analyse de chacun de ces sous-procès fondamentaux ${ }^{82}$.

\section{c. Complexités horizontales et verticales}

La tentation est grande de postuler une structure de domination entre ces sous-procès de manière à reconstruire l'évolution du grand jeu comme si elle n'était guidée que par les impératifs d'un seul sousprocès moteur. Un tel procédé de réduction nous paraît dangereux: il pourrait bien escamoter les complexités mentionnées à la fin de la section sur l'historiographie canadienne. Il nous semble plutôt que c'est par la combinaison et l'intégration des divers sous-procès qu'il sera possible de discerner les contours et les fondements des divers secteurs, régions, sections, etc. à la surface de la socio-économie.

81 Le sous-procès démographique constitue un exemple simple: les démographes en ont étudié le fonctionnement et ont atteint un fort degré de formalisation. Les analyses ont repéré les rythmes de reproduction des populations et les règles qui définissent les structures complexes du stock de population d'une génération à l'autre. Les résultats dans ce cas sont évidemment plus impressionnants que ceux auxquels on a pu arriver jusqu'ici dans la définition de génotypes pour la reproduction des groupes sociaux; mais là aussi les travaux importants se multiplient sur la reproduction des structures sociales.

${ }_{82}$ À propos du sous-procès démographique comme des autres, voir les études intéressantes mentionnées dans les notes $41,42,43$ et 66 . Le sous-procès production-échange a fait l'objet de nombreux travaux de nature sociographique scrutant différents aspects de l'expérience canadienne; mais on trouvera très peu d'études systématiques sur l'ensemble du XIXe siècle. On pourrait cependant mentionner les travaux bien connus de Aitken, de Dubuc, de Tulchinsky, de Gilmour, de Langdon, de Naylor (et de la réaction critique de Macdonald). À propos du sous-procès financier, on peut ajouter E.P. Neufeld, The Financial System of Canada (Macmillan, 1972) et des sections du livre de Bond et Shearer, The Economics of the Canadian Financial System, (Scarborough, 1972). Sur l'écologie des groupes et leurs motivations, on peut recourir à un certain nombre d'études des classes ou des élites ou de diverses stratifications sociales dès les débuts du XIXe siècle (les livres de F. Ouellet, les études du GRSM, notre "Groupes sociaux et pouvoir...", la spectrographie de Toronto à l'époque victorienne par Goheen, celle de Winnipeg par Artibise, etc. (voir notes $41 \mathrm{ss})$. Sur le sous-procès étatique, là encore le travail se révèle surtout sociographique et fragmentaire, avec des études par Acheson, Nelles, Aitken, Paquet et Wallot, etc. Enfin, l'historiographie a démarré sur le sous-procès de répartition (v.g. les études de Bliss) et W.I. Gillespie dirige diverses enquêtes sur cette question à l'université Carleton. Pour un survol de la période après 1867, voir O.J. Firestone, Canada's Economic Development 1867-1953, (Londres, 1958). On peut repérer de nombreuses références dans T.J.O. Dick, Economic History of Canada. A Guide to Information Sources (Détroit, 1978), en particulier les chap. III et IV. Il est évident que dans le cas de l'étude du Canada comme socio-économie petite, ouverte, dépendante et balkanisée, l'importance de l'impact des forces exogènes ne saurait être ignorée. Il faut donc percevoir la socio-économie canadienne du XIXe siècle comme insérée à tout moment dans un procès socio-économique mondial qui entraîne sur le Canada des impacts asymétriques et des emprises de structures. Ces considérations bannissent l'étude des sous-jeux en vase clos. 
On peut imaginer que la complexité horizontale et verticale du procès socio-économique canadien au XIXe siècle ne se révélera qu'à travers la superposition de ces sous-procès comme autant de "transparents" composant dans leur lacis le détail de secteurs signifiants et de périodes pertinentes - secteurs caractérisés par un mểme tonus, des réactions et ajustements de même type, périodes démarquées par des discontinuités dans le mode de fonctionnement dans le temps ${ }^{83}$.

Akerman a cherché à construire un tel tableau dans toute sa complexité horizontale (secteurs) et verticale (périodes): les mots mêmes et les catégories d'Akerman annoncent les caractères de cette histoire générale et de ce tableau que nous évoquions à propos des travaux de la troisième génération des Annales $^{84}$. Plus récemment, Gillard a suggéré des techniques utilisant les données disponibles pour diviser la réalité selon des procès combinés ${ }^{85}$.

Ces procédés et bien d'autres inspirés par les premiers résultats des travaux de méso-analyse, permettent d'espérer la reconstruction éventuelle de l'expérience du Canada au XIXe siècle à partir des sous-procès qui découperaient les agrégats fonctionnels d'opération (qui peuvent fort bien différer des unités de surface comme les régions géo-politiques) et les cassures profondes dans l'expérience de cette socio-économie (qui peuvent fort bien n'être pas repérables en fonction des césures traditionnelles de l'historiographie).

La composition des sous-procès peut donc expliquer pourquoi le procès socio-économique canadien s'est institué différemment de place en place et de période en période au XIXe siècle. Plus encore, elle peut faire apparaître les sources et les causes de certains métaprocès comme l'urbanisation et l'industrialisation (donc fournir certains éclaircissements sur leur dynamique). Ceux-ci transcendent la socio-économie canadienne, mais s'accomplissent ici d'une façon particulière à cause justement de la trame institutionnelle de la socio-économie et de son tonus propre ${ }^{86}$.

83 L'analogie des "transparents" peut-être utile, mais elle est boiteuse. En effet, les "transparents" qu'on superpose sont indépendants les uns des autres et statiques, alors que les sous-procès sont en interaction et intercréation constante et fondamentalement dynamiques, c'est-à-dire en action.

84 J. Akerman, op. cit., chap. I, surtout section 2.

85 L. Gillard, "Premier bilan d'une recherche économique sur la méso-analyse", Revue économique, 3 (1975): 504ss.

86 Il est évident que ces analyses n'ambitionnent pas de fournir une explication exhaustive et complète. Celle-ci devra faire la part des "intrusions" en provenance du monde extérieur. 


\section{CONCLUSION}

Pour que s'accomplisse une telle reconstruction rationnelle du XIXe siècle canadien, il reste évidemment beaucoup de travail théorique et empirique à faire. D'ailleurs, la problématique esquissée plus haut ne peut servir de panacée ou de talisman: elle aiguille simplement sur des directions de recherche et contribue à éclairer certaines limites d'études partielles qui se sont déguisées en études générales. De plus, elle peut expliquer pourquoi l'examen de certains méta-procès comme l'urbanisation et l'industrialisation n'a progressé que très lentement: de telles fouilles ont pu s'avérer prématurées dans la mesure où faisait défaut l'analyse des sousprocès fondamentaux qui conjointement les expliquent.

Dans le présent article, nous avons voulu tout au plus établir les raisons pour lesquelles un pari sur la méso-histoire nous paraît fondé et montrer comment ce pari s'inscrit en continuité avec les travaux de la dernière décennie sur le chantier historique. La problématique suggérée revêt une forme préliminaire et la stratégie de recherche qu'elle implique peut paraître ambitieuse. Guidée par elle, certains travaux en cours nous donnent à penser que nous n'avons pas surestimé son pouvoir heuristique ${ }^{87}$. Comme dirait Macbeath, "our hopes are as good as your fears".

87 Nous avons utilisé cet appareil dans deux travaux récents: "Sur quelques discontinuités dans l'expérience socio-économique québécoise: une hypothèse" (miméo, 1979); "Canadian Cities as Social Technologies: An Exploratory Essay", communication au 43e congrès international des Américanistes, Vancouver, 11-17 août 1979, à paraître dans les Actes du colloque en 1980. 